\title{
Preference Attitude-Based Method for Ranking Intuitionistic Fuzzy Numbers and Its Application in Renewable Energy Selection
}

\author{
Jian Lin, ${ }^{1,2}$ Fanyong Meng $\mathbb{D}^{3}{ }^{3}$ Riqing Chen $\mathbb{D}^{1},{ }^{1}$ and Qiang Zhang ${ }^{4}$ \\ ${ }^{1}$ College of Computer and Information, Fujian Agriculture and Forestry University, Fuzhou 350002, China \\ ${ }^{2}$ Institute of Big Data for Agriculture and Forestry, Fujian Agriculture and Forestry University, Fuzhou 350002, China \\ ${ }^{3}$ School of Business, Central South University, Changsha 410083, China \\ ${ }^{4}$ School of Management and Economics, Beijing Institute of Technology, Beijing 100081, China
}

Correspondence should be addressed to Riqing Chen; riqing.chen@fafu.edu.cn

Received 19 September 2017; Accepted 16 January 2018; Published 22 February 2018

Academic Editor: Sigurdur F. Hafstein

Copyright (C) 2018 Jian Lin et al. This is an open access article distributed under the Creative Commons Attribution License, which permits unrestricted use, distribution, and reproduction in any medium, provided the original work is properly cited.

\begin{abstract}
Many applications of intuitionistic fuzzy sets depend on ranking or comparing intuitionistic fuzzy numbers. This paper presents a novel ranking method for intuitionistic fuzzy numbers based on the preference attitudinal accuracy and score functions. The proposed ranking method considers not only the preference attitude of decision maker, but also all the possible values in feasible domain. Some desirable properties of preference attitudinal accuracy and score functions are verified in detail. A total order on the set of intuitionistic fuzzy numbers is established by using the proposed two functions. The proposed ranking method is also applied to select renewable energy. The advantage and validity of the proposed method are shown by comparing with some representative ranking methods.
\end{abstract}

\section{Introduction}

Atanassov [1] introduced the concept of intuitionistic fuzzy sets characterized by a membership function, a nonmembership function, and a hesitancy function. Due to the increasing complexity of real life problems, intuitionistic fuzzy set is very suitable for representing fuzzy information under complicated and uncertain settings as an extension of traditional fuzzy set. Intuitionistic fuzzy set theory has been deeply discussed by many scholars since the notations appearance and applied in various fields, such as decision-making [2-7], supplier selection [8-10], pattern recognition [11-14], medical diagnosis [15, 16], and artificial intelligence [17, 18].

Many applications of intuitionistic fuzzy sets depend on ranking or comparing intuitionistic fuzzy numbers. A number of researchers focus on the order relation of intuitionistic fuzzy numbers over the past two decades. Chen and Tan [19] proposed a score function to evaluate the score of intuitionistic fuzzy values. Through analysis on the limitation of Chen and Tan's score function, Hong and Choi [20] improved their ranking method by adding an accuracy function. $\mathrm{Xu}[21]$ gave a kind of order relation to rank the intuitionistic fuzzy numbers by combining the score and accuracy function. Wang et al. [22] introduced a novel score function to measure the degree of suitability. Some desirable properties of the novel score function were discussed. Ye [23] developed an improved algorithm for score functions based on hesitancy degree. By using the intuitionistic fuzzy point operators, Liu and Wang [24] defined a series of new score functions for dealing with multicriteria decision-making problems. Jafarian and Rezvani [25] presented a method for mapping the intuitionistic fuzzy numbers into the crisp values and described the concept of spread value of intuitionistic fuzzy number. To analyze the fuzzy meaning of an intuitionistic fuzzy value, Yu et al. [26] formalized an intuitionistic fuzzy value as a fuzzy subset and determined the dominance relation between two intuitionistic fuzzy values. Guo [27] built a new ranking model based on the viewpoint of amount of information. A total order which extended the usual partial order was analyzed in deep. Zhang and $\mathrm{Xu}$ [28] used a 
special function to define the order of intuitionistic fuzzy numbers. Some good mathematical properties on algebraic intuitionistic fuzzy numbers were also given. Lakshmana et al. [29] derived a total order on the entire class of intuitionistic fuzzy number by applying upper lower dense sequence to the interval. Gupta et al. [30] utilized relative comparisons based on the advantage and disadvantage scores of intuitionistic fuzzy numbers. The relative comparison of intuitionistic fuzzy numbers took the membership, nonmembership, and hesitancy degree into account. Xing et al. [31] proposed an Euclidean distance-based approach to ranking intuitionistic fuzzy numbers. However, the above-mentioned methods for ranking intuitionistic fuzzy numbers do not consider the risk attitude of decision maker which is very flexible and useful in real-world applications. A valid ranking method should be established in accordance with the preference attitude of decision maker. To do this, Chen [32] conducted a comparative analysis of score functions for ranking intuitionistic fuzzy numbers. A parameterized score function was developed to represent a mixed result of positive and negative outcome expectations. Wang et al. [33] proposed a new score function based on relative entropy. The risk attitudes of decision makers were defined by risk preference indexes. Wan et al. [34] utilized the closeness degree to characterize the amount of information based on TOPSIS method. A novel risk attitudinal ranking measure is developed to rank the intuitionistic fuzzy numbers. Nevertheless, the above preference attitudebased ranking methods just focus on the extreme points of feasible domain; the other possible values in feasible domain are ignored. Therefore, these ranking methods may lose some valuable information, which can be useful in determining the order relation of intuitionistic fuzzy numbers. To overcome the shortages of existing ranking methods, this paper proposes a preference attitudinal method for ranking intuitionistic fuzzy numbers based on preference attitudinal accuracy and score functions. The proposed ranking method considers not only the preference attitude of decision maker, but also all the possible values in feasible domain. Some desirable properties of preference attitudinal accuracy and score functions are discussed. Moreover, the proposed method is applied to deal with renewable energy selection problem. The advantage and validity of the proposed method are shown in detail by comparing with some representative ranking methods.

The rest of this paper is structured as follows. Section 2 introduces some basic concepts on intuitionistic fuzzy set. In Section 3, the preference attitudinal accuracy and score functions of intuitionistic fuzzy numbers are proposed. Section 4 presents the order relation between intuitionistic fuzzy numbers. Numeral examples and comparison are shown in Section 5 .

\section{Preliminaries}

In the following, some basic concepts on intuitionistic fuzzy set are introduced to facilitate future discussions.

Definition 1 (see [1]). Let $X$ be a universe of discourse. An intuitionistic fuzzy set $A$ over $X$ is expressed as

$$
A=\left\{\left\langle x, \mu_{A}(x), v_{A}(x)\right\rangle \mid x \in X\right\},
$$

where $\mu_{A}(x)$ and $v_{A}(x)$ are the membership degree and nonmembership degree of $x$ to $A$, respectively, such that $\mu_{A}(x), v_{A}(x) \in[0,1], \mu_{A}(x)+v_{A}(x) \leq 1$. The hesitation degree of $x$ to $A$ is denoted by $\pi_{A}(x)=1-\mu_{A}(x)-v_{A}(x)$.

Obviously, for each $x \in X$, we have $\pi_{A}(x) \in[0,1]$. For simplicity, $\alpha=(\mu, v)$ is called an intuitionistic fuzzy number (IFN), such that $\mu, v \in[0,1], \mu+v \leq 1$, and $\pi(\alpha)=1-\mu-v$. The set of all IFNs is denoted by IFN.

Let $\alpha_{1}=\left(\mu_{1}, v_{1}\right)$ and $\alpha_{2}=\left(\mu_{2}, v_{2}\right)$ be two IFNs. Clearly, $\alpha_{1}=\alpha_{2}$ if and only if $\mu_{1}=\mu_{2}$ and $v_{1}=v_{2}$. Based on the score function [19] and accuracy function [20], Xu [21] introduced the operational laws and ordering relation among intuitionistic fuzzy numbers as follows.

Definition 2. Let $\alpha_{1}=\left(\mu_{1}, v_{1}\right)$ and $\alpha_{2}=\left(\mu_{2}, v_{2}\right)$ be two IFNs; then

$$
\begin{aligned}
& \text { (1) } \alpha_{1} \oplus \alpha_{2}=\left(\mu_{1}+\mu_{2}-\mu_{1} \mu_{2}, v_{1} v_{2}\right), \\
& \text { (2) } k \otimes \alpha_{1}=\left(1-\left(1-\mu_{1}\right)^{k}, v_{1}{ }^{k}\right), k>0 .
\end{aligned}
$$

Definition 3. Let $\alpha=(\mu, v)$ be an IFN. The accuracy and score function of $\alpha$ are, respectively, represented by

$$
\begin{aligned}
& A_{X}(\alpha)=\mu+v, \\
& S_{X}(\alpha)=\mu-v .
\end{aligned}
$$

It is clear that $A_{X}(\alpha) \in[0,1]$ and $S_{X}(\alpha) \in[-1,1]$. From (2) and (3), we have $A_{X}(\alpha)=S_{X}(\alpha)+2 v$. Thus, $A_{X}(\alpha) \geq S_{X}(\alpha)$ holds.

Definition 4. Let $\alpha_{1}=\left(\mu_{1}, v_{1}\right)$ and $\alpha_{2}=\left(\mu_{2}, v_{2}\right)$ be two IFNs. The ordering relation is established as follows:

(1) If $S_{X}\left(\alpha_{1}\right)<S_{X}\left(\alpha_{2}\right)$, then $\alpha_{1} \prec_{X} \alpha_{2}$.

(2) If $S_{X}\left(\alpha_{1}\right)=S_{X}\left(\alpha_{2}\right)$, then

(a) if $A_{X}\left(\alpha_{1}\right)=A_{X}\left(\alpha_{2}\right)$, then $\alpha_{1}=\alpha_{2}$,

(b) if $A_{X}\left(\alpha_{1}\right)<A_{X}\left(\alpha_{2}\right)$, then $\alpha_{1} \prec_{X} \alpha_{2}$.

\section{The Preference Attitudinal Accuracy and Score Functions of Intuitionistic Fuzzy Numbers}

In this section, we will propose the definitions of preference attitudinal accuracy and score functions by adding an attitudinal parameter in double integral.

\subsection{The Preference Attitudinal Accuracy Function}

Definition 5. Let $\alpha=(\mu, v)$ be an IFN. The feasible domain of $\alpha$ is represented by

$$
\begin{aligned}
D_{\alpha} & =\{(x, y) \mid \mu \leq x \leq \mu+\pi, v \leq y \leq v+\pi, x+y \\
& \leq 1\}
\end{aligned}
$$

where $\pi=1-u-v$. By considering the hesitation degree of $\alpha, D_{\alpha}$ denotes the set of feasible intuitionistic fuzzy numbers 


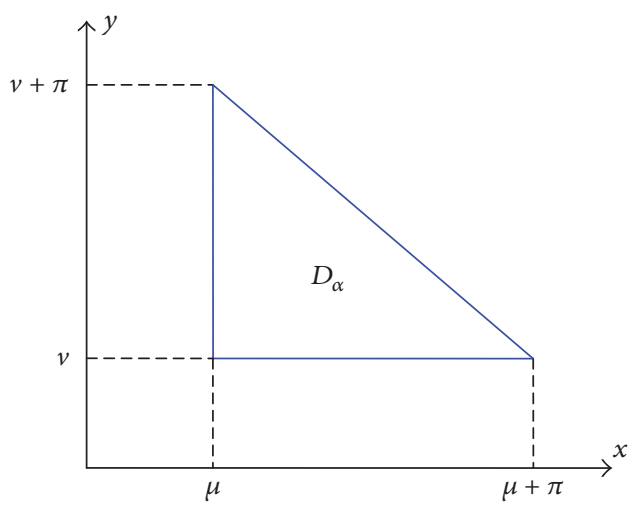

Figure 1: The feasible domain with respect to $\alpha$.

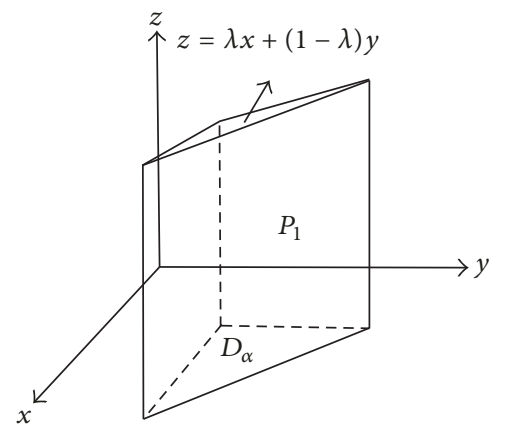

FIgURE 2: The graph of oblique triangular prism $P_{1}$.

with respect to $\alpha$. The feasible domain $D_{\alpha}$ is illustrated in Figure 1.

Definition 6. Let $\alpha=(\mu, v)$ be an IFN. The preference attitudinal accuracy function of $\alpha$ is defined by

$$
A_{\lambda}(\alpha)=\frac{1}{\operatorname{area}\left(D_{\alpha}\right)} \iint_{D_{\alpha}}(\lambda x+(1-\lambda) y) d x d y,
$$

where $D_{\alpha}$ is the feasible domain of intuitionistic fuzzy number $\alpha$ and area $\left(D_{\alpha}\right)$ is the area of feasible domain $D_{\alpha}$. The parameter $\lambda$ can be regarded as the level of decision maker or assessor's preference attitude towards positive information, $\lambda \in[0,1]$. In particular, decision maker or assessor is said to be neutral to the positive and negative information when $\lambda=0.5$.

From (5), $A_{\lambda}(\alpha)$ represents the average value of accuracy function over feasible domain $D_{\alpha} \cdot \iint_{D_{\alpha}}(\lambda x+(1-\lambda) y) d x d y$ is equal to the volume of the oblique triangular prism $P_{1}$ shown in Figure 2. It means that $A_{\lambda}(\alpha)$ can be regarded as the average height of oblique triangular prism $P_{1}$. By Definition 5, we have

$$
\operatorname{area}\left(D_{\alpha}\right)=\frac{1}{2} \pi^{2}=\frac{1}{2}(1-\mu-v)^{2}
$$

Since

$$
\begin{aligned}
& \iint_{D_{\alpha}}(\lambda x+(1-\lambda) y) d x d y \\
& =\int_{\mu}^{1-v}\left(\int_{v}^{1-x}(\lambda x+(1-\lambda) y) d y\right) d x \\
& =\frac{1}{6}(1-\mu-v)^{2}(1-\mu+2 v) \\
& \quad+\frac{\lambda}{2}(1-\mu-v)^{2}(\mu-v),
\end{aligned}
$$

we get the following expression of preference attitudinal accuracy function

$$
A_{\lambda}(\alpha)=\frac{1-\mu+2 v}{3}+\lambda(\mu-v)
$$

which can be equivalently written as

$$
A_{\lambda}(\alpha)=\lambda \frac{2 \mu-v+1}{3}+(1-\lambda) \frac{2 v-\mu+1}{3} .
$$

The preference attitudinal accuracy function satisfies the following desirable properties.

Property 7. Let $\alpha=(\mu, v)$ be an IFN. For each parameter $\lambda \epsilon$ $[0,1]$, we have

$$
\begin{aligned}
0 & \leq \min \left\{\frac{2 \mu-v+1}{3}, \frac{2 v-\mu+1}{3}\right\} \leq A_{\lambda}(\alpha) \\
& \leq \max \left\{\frac{2 \mu-v+1}{3}, \frac{2 v-\mu+1}{3}\right\} \leq 1 .
\end{aligned}
$$

Proof. Property 7 can be proved in two cases, shown as follows:

(1) If $\mu \geq v$, then we have $0 \leq 2 v-\mu+1 \leq 2 \mu-v+1 \leq 3$. For all $\lambda \in[0,1]$, it holds that

$$
\begin{aligned}
\frac{2 v-\mu+1}{3} & \leq \lambda \frac{2 \mu-v+1}{3}+(1-\lambda) \frac{2 v-\mu+1}{3} \\
& \leq \frac{2 \mu-v+1}{3} .
\end{aligned}
$$

Since $(2 v-\mu+1) / 3 \geq 0$ and $(2 \mu-v+1) / 3 \leq 1$, by (8), we have

$$
0 \leq \frac{2 v-\mu+1}{3} \leq A_{\lambda}(\alpha) \leq \frac{2 \mu-v+1}{3} \leq 1 .
$$

(2) If $\mu<v$, then we have $0 \leq 2 \mu-v+1<2 v-\mu+1 \leq 3$. For all $\lambda \in[0,1]$, it holds that

$$
\begin{aligned}
\frac{2 \mu-v+1}{3} & \leq \lambda \frac{2 \mu-v+1}{3}+(1-\lambda) \frac{2 v-\mu+1}{3} \\
& \leq \frac{2 v-\mu+1}{3} .
\end{aligned}
$$

Since $(2 v-\mu+1) / 3 \leq 1$ and $(2 \mu-v+1) / 3 \geq 0$, by (8), we have

$$
0 \leq \frac{2 \mu-v+1}{3} \leq A_{\lambda}(\alpha) \leq \frac{2 v-\mu+1}{3} \leq 1 .
$$

In sum, Property 7 is proved. 
Property 8. Let $\alpha_{1}=\left(\mu_{1}, v_{1}\right)$ and $\alpha_{2}=\left(\mu_{2}, v_{2}\right)$ be two IFNs. If $v_{1}-v_{2} \leq \mu_{1}-\mu_{2} \leq 2\left(v_{1}-v_{2}\right)$, then $A_{\lambda}\left(\alpha_{1}\right) \geq A_{\lambda}\left(\alpha_{2}\right)$ holds for all $\lambda \in[0,1]$.

Proof. From (8), we have

$$
\begin{aligned}
& A_{\lambda}\left(\alpha_{1}\right)=\frac{1-\mu_{1}+2 v_{1}}{3}+\lambda\left(\mu_{1}-v_{1}\right), \\
& A_{\lambda}\left(\alpha_{2}\right)=\frac{1-\mu_{2}+2 v_{2}}{3}+\lambda\left(\mu_{2}-v_{2}\right) .
\end{aligned}
$$

It follows that

$$
\begin{aligned}
A_{\lambda}\left(\alpha_{1}\right)-A_{\lambda}\left(\alpha_{2}\right)= & \frac{1}{3}\left(\mu_{2}-\mu_{1}+2\left(v_{1}-v_{2}\right)\right) \\
& +\lambda\left(\mu_{1}-\mu_{2}+v_{2}-v_{1}\right) .
\end{aligned}
$$

Since $v_{1}-v_{2} \leq \mu_{1}-\mu_{2} \leq 2\left(v_{1}-v_{2}\right)$ and $0 \leq \lambda \leq 1$, we have $A_{\lambda}\left(\alpha_{1}\right)-A_{\lambda}\left(\alpha_{2}\right) \geq 0$.

This completes the proof of Property 8.

Property 9. For any two IFNs $\alpha_{1}=\left(\mu_{1}, v_{1}\right)$ and $\alpha_{2}=\left(\mu_{2}, v_{2}\right)$, $A_{0.5}\left(\alpha_{1}\right)>A_{0.5}\left(\alpha_{2}\right)$ if and only if $A_{X}\left(\alpha_{1}\right)>A_{X}\left(\alpha_{2}\right)$.

Proof. When $\lambda=0.5$, we have $A_{0.5}\left(\alpha_{i}\right)=\left(\mu_{i}+v_{i}+2\right) / 6$, $i=1,2$. Based on (8), we get $A_{0.5}\left(\alpha_{i}\right)=(1 / 6) A_{X}\left(\alpha_{i}\right)+1 / 3, i=$ 1,2 . Thus, $A_{X}\left(\alpha_{1}\right)>A_{X}\left(\alpha_{2}\right)$ can be equivalently expressed as

$$
\begin{aligned}
A_{0.5}\left(\alpha_{1}\right) & =\frac{1}{6} A_{X}\left(\alpha_{1}\right)+\frac{1}{3}>\frac{1}{6} A_{X}\left(\alpha_{2}\right)+\frac{1}{3} \\
& =A_{0.5}\left(\alpha_{2}\right) .
\end{aligned}
$$

This completes the proof of Property 9.

Property 10. Let $\alpha=(\mu, v)$ be an IFN. $\bar{\alpha}=(\nu, \mu)$ is the complementary intuitionistic fuzzy number of $\alpha$. For each attitudinal parameter $\lambda \in[0,1]$, we have $A_{\lambda}(\alpha)=A_{1-\lambda}(\bar{\alpha})$.

Proof. From (9), it follows that

$$
\begin{aligned}
A_{1-\lambda}(\bar{\alpha}) & =(1-\lambda) \frac{2 v-\mu+1}{3}+\lambda \frac{2 \mu-v+1}{3} \\
& =A_{\lambda}(\alpha) .
\end{aligned}
$$

Thus, Property 10 is proved.

Property 11 (sensitivity analysis). Given two IFNs $\alpha_{1}=$ $\left(\mu_{1}, v_{1}\right)$ and $\alpha_{2}=\left(\mu_{2}, v_{2}\right)$. Let $\lambda$ be the attitudinal parameter under which it has satisfied the fact that $A_{\lambda}\left(\alpha_{1}\right) \leq A_{\lambda}\left(\alpha_{2}\right)$. Let $\Delta \lambda$ be a perturbation of the attitudinal parameter $\lambda$ with
$\lambda+\Delta \lambda \in[0,1]$. Then, $A_{\lambda+\Delta \lambda}\left(\alpha_{1}\right) \leq A_{\lambda+\Delta \lambda}\left(\alpha_{2}\right)$ holds if and only if

$$
\begin{gathered}
-\lambda \leq \Delta \lambda \leq \min \left\{\frac{A_{\lambda}\left(\alpha_{2}\right)-A_{\lambda}\left(\alpha_{1}\right)}{\mu_{1}-\mu_{2}+v_{2}-v_{1}}, 1-\lambda\right\}, \\
\mu_{1}-\mu_{2}>v_{1}-v_{2} ; \\
\max \left\{\frac{A_{\lambda}\left(\alpha_{2}\right)-A_{\lambda}\left(\alpha_{1}\right)}{\mu_{1}-\mu_{2}+v_{2}-v_{1}},-\lambda\right\} \leq \Delta \lambda \leq 1-\lambda, \\
\quad \mu_{1}-\mu_{2}<v_{1}-v_{2} ; \\
-\lambda \leq \Delta \lambda \leq 1-\lambda, \quad \mu_{1}-\mu_{2}=v_{1}-v_{2} .
\end{gathered}
$$

Proof. Since $0 \leq \lambda+\Delta \lambda \leq 1$, we have $-\lambda \leq \Delta \lambda \leq 1-\lambda$. Considering of

$$
\begin{aligned}
& A_{\lambda+\Delta \lambda}\left(\alpha_{1}\right)=A_{\lambda}\left(\alpha_{1}\right)+\Delta \lambda\left(\mu_{1}-v_{1}\right), \\
& A_{\lambda+\Delta \lambda}\left(\alpha_{2}\right)=A_{\lambda}\left(\alpha_{2}\right)+\Delta \lambda\left(\mu_{2}-v_{2}\right),
\end{aligned}
$$

the following inequalities are equivalent:

$$
\begin{gathered}
A_{\lambda+\Delta \lambda}\left(\alpha_{1}\right) \leq A_{\lambda+\Delta \lambda}\left(\alpha_{2}\right) \Longleftrightarrow \\
A_{\lambda}\left(\alpha_{2}\right)-A_{\lambda}\left(\alpha_{1}\right) \geq \Delta \lambda\left(\mu_{1}+v_{2}-\mu_{2}-v_{1}\right) .
\end{gathered}
$$

We can consider (21) in three cases, shown as follows:

(a) If $\mu_{1}-\mu_{2}=v_{1}-v_{2}$, then inequalities $A_{\lambda+\Delta \lambda}\left(\alpha_{1}\right) \leq$ $A_{\lambda+\Delta \lambda}\left(\alpha_{2}\right)$ and $A_{\lambda}\left(\alpha_{2}\right)-A_{\lambda}\left(\alpha_{1}\right) \geq 0$ are equivalent.

It means that (21) is true for all $\Delta \lambda$ with $-\lambda \leq \Delta \lambda \leq 1-\lambda$.

(b) If $\mu_{1}-\mu_{2}>v_{1}-v_{2}$, then

$$
\begin{aligned}
A_{\lambda+\Delta \lambda}\left(\alpha_{1}\right) & \leq A_{\lambda+\Delta \lambda}\left(\alpha_{2}\right) \Longleftrightarrow \\
\Delta \lambda & \leq \frac{A_{\lambda}\left(\alpha_{2}\right)-A_{\lambda}\left(\alpha_{1}\right)}{\mu_{1}-\mu_{2}+v_{2}-v_{1}} .
\end{aligned}
$$

Notice that $\Delta \lambda$ is subject to the constraint $-\lambda \leq \Delta \lambda \leq 1-\lambda$; inequality $A_{\lambda+\Delta \lambda}\left(\alpha_{1}\right) \leq A_{\lambda+\Delta \lambda}\left(\alpha_{2}\right)$ is equivalent to

$$
-\lambda \leq \Delta \lambda \leq \min \left\{\frac{A_{\lambda}\left(\alpha_{2}\right)-A_{\lambda}\left(\alpha_{1}\right)}{\mu_{1}-\mu_{2}+v_{2}-v_{1}}, 1-\lambda\right\} .
$$

(c) If $\mu_{1}-\mu_{2}<v_{1}-v_{2}$, then

$$
\begin{aligned}
A_{\lambda+\Delta \lambda}\left(\alpha_{1}\right) & \leq A_{\lambda+\Delta \lambda}\left(\alpha_{2}\right) \Longleftrightarrow \\
\Delta \lambda & \geq \frac{A_{\lambda}\left(\alpha_{2}\right)-A_{\lambda}\left(\alpha_{1}\right)}{\mu_{1}-\mu_{2}+v_{2}-v_{1}} .
\end{aligned}
$$

Therefore, inequality $A_{\lambda+\Delta \lambda}\left(\alpha_{1}\right) \leq A_{\lambda+\Delta \lambda}\left(\alpha_{2}\right)$ is equivalent to

$$
\max \left\{\frac{A_{\lambda}\left(\alpha_{2}\right)-A_{\lambda}\left(\alpha_{1}\right)}{\mu_{1}-\mu_{2}+v_{2}-v_{1}},-\lambda\right\} \leq \Delta \lambda \leq 1-\lambda .
$$

In sum, Property 11 is proved.

Remark 12. As everyone knows, it is very difficult to determine the exact value of parameter $\lambda$. To deal with this problem, Table 1 is presented for estimating the appropriate value of parameter $\lambda$. 
TABLE 1: Preference attitude towards information and the corresponding value of $\lambda$.

\begin{tabular}{lc}
\hline The assessor's preference attitude towards information & The value of $\lambda$ \\
\hline The negative information is absolutely preferred (NA) & 0 \\
The negative information is extremely preferred (NE) & 0.1 \\
The negative information is strongly preferred (NST) & 0.2 \\
The negative information is moderately preferred (NM) & 0.3 \\
The negative information is slightly preferred (NSL) & 0.4 \\
The assessor is neutral to the positive and negative information (NPN) & 0.5 \\
The positive information is slightly preferred (PSL) & 0.6 \\
The positive information is moderately preferred (PM) & 0.7 \\
The positive information is strongly preferred (PST) & 0.8 \\
The positive information is extremely preferred (PE) & 0.9 \\
The positive information is absolutely preferred (PA) & 1 \\
\hline
\end{tabular}

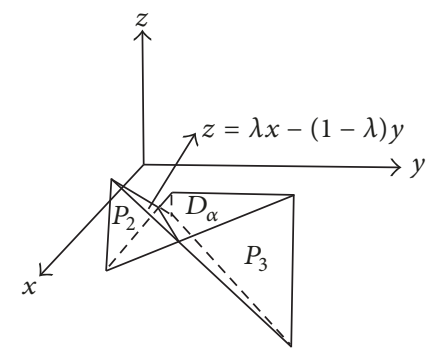

Figure 3: The graphs of cubic $P_{2}$ and $P_{3}$.

It is clear that Table 1 proposed some discrete values of parameter $\lambda$ corresponding to the linear preference of assessor. If the preference of assessor is nonlinear and the value of $\lambda$ is continuous, some nonlinear functions can be used here to measure the value of $\lambda$ : that is, $f: \Omega \rightarrow[0,1]$ such that $f(\mathrm{NA})=0, f(\mathrm{NPN})=0.5$ and $f(\mathrm{PA})=1 . \Omega$ is the set of assessor's preference attitudes towards information.

3.2. The Preference Attitudinal Score Function. Similar to the preference attitudinal accuracy function, the preference attitudinal score function can be defined as follows.

Definition 13. Let $\alpha=(\mu, v)$ be an IFN. The preference attitudinal score function of $\alpha$ is defined by

$$
S_{\lambda}(\alpha)=\frac{1}{\operatorname{area}\left(D_{\alpha}\right)} \iint_{D_{\alpha}}(\lambda x-(1-\lambda) y) d x d y,
$$

where $D_{\alpha}$ is the feasible domain of intuitionistic fuzzy number $\alpha$ and area $\left(D_{\alpha}\right)$ is the area of feasible domain $D_{\alpha}$.

From (26), $S_{\lambda}(\alpha)$ represents the average value of score function over feasible domain $D_{\alpha}$. Denote the volume of $P_{2}$ by $V_{\text {up }}$ and the volume of $P_{3}$ by $V_{\text {below }}$, respectively. Then, we have $\iint_{D_{\alpha}}(\lambda x-(1-\lambda) y) d x d y=V_{\text {up }}-V_{\text {below }}$. The graphs of cubic $P_{2}$ and $P_{3}$ are illustrated in Figure 3. Accordingly, $S_{\lambda}(\alpha)$ can be regarded as the average values of variable $z$ over feasible domain $D_{\alpha}$. Since

$$
\begin{aligned}
& \iint_{D_{\alpha}}(\lambda x-(1-\lambda) y) d x d y \\
& \quad=\int_{\mu}^{1-v}\left(\int_{v}^{1-x}(\lambda x-(1-\lambda) y) d y\right) d x \\
& =\frac{1}{6}(1-\mu-v)^{2}(\mu-2 v-1) \\
& \quad+\frac{\lambda}{6}(1-\mu-v)^{2}(\mu+v+2),
\end{aligned}
$$

based on (6) and (26), the following expression of preference attitudinal score function is derived:

$$
S_{\lambda}(\alpha)=\frac{\mu-2 v-1}{3}+\frac{\lambda}{3}(\mu+v+2)
$$

which can be equivalently written as

$$
S_{\lambda}(\alpha)=\lambda \frac{2 \mu-v+1}{3}+(1-\lambda) \frac{\mu-2 v-1}{3} .
$$

The preference attitudinal score function satisfies the following desirable properties.

Property 14. Let $\alpha_{1}=\left(\mu_{1}, v_{1}\right)$ and $\alpha_{2}=\left(\mu_{2}, v_{2}\right)$ be two IFNs. If $\mu_{1} \geq \mu_{2}$ and $v_{1} \leq v_{2}$, then $S_{\lambda}\left(\alpha_{1}\right) \geq S_{\lambda}\left(\alpha_{2}\right)$ holds for all $\lambda \in[0,1]$.

Proof. From (28), we have

$$
\begin{aligned}
& S_{\lambda}\left(\alpha_{1}\right)=\frac{\mu_{1}-2 v_{1}-1}{3}+\frac{\lambda}{3}\left(\mu_{1}+v_{1}+2\right), \\
& S_{\lambda}\left(\alpha_{2}\right)=\frac{\mu_{2}-2 v_{2}-1}{3}+\frac{\lambda}{3}\left(\mu_{2}+v_{2}+2\right) .
\end{aligned}
$$

It follows that

$$
\begin{aligned}
S_{\lambda}\left(\alpha_{1}\right)-S_{\lambda}\left(\alpha_{2}\right) \\
=\frac{\lambda}{3}\left(2\left(\mu_{1}-\mu_{2}\right)+v_{2}-v_{1}\right) \\
\quad+\frac{(1-\lambda)}{3}\left(\mu_{1}-\mu_{2}+2\left(v_{2}-v_{1}\right)\right) .
\end{aligned}
$$


Since $\mu_{1} \geq \mu_{2}, v_{1} \leq v_{2}$, and $0 \leq \lambda \leq 1$, we have $S_{\lambda}\left(\alpha_{1}\right)-$ $S_{\lambda}\left(\alpha_{2}\right) \geq 0$.

Thus, Property 14 is proved.

Property 15. Let $\alpha=(\mu, v)$ be an IFN. The preference attitudinal score function $S_{\lambda}(\alpha)$ is monotonic with respect to the parameter $\lambda$; that is, if $\lambda_{1} \geq \lambda_{2}$, then $S_{\lambda_{1}}(\alpha) \geq S_{\lambda_{2}}(\alpha)$.

Proof. From (28), we have

$$
\begin{aligned}
& S_{\lambda_{1}}(\alpha)=\frac{\mu-2 v-1}{3}+\frac{\lambda_{1}}{3}(\mu+v+2), \\
& S_{\lambda_{2}}(\alpha)=\frac{\mu-2 v-1}{3}+\frac{\lambda_{2}}{3}(\mu+v+2) .
\end{aligned}
$$

Thus, we have $S_{\lambda_{1}}(\alpha)-S_{\lambda_{2}}(\alpha)=(1 / 3)\left(\lambda_{1}-\lambda_{2}\right)(\mu+v+2) \geq 0$.

This completes the proof of Property 15.

Property 16. Let $\alpha=(\mu, v)$ be an IFN. For each parameter $\lambda \epsilon$ $[0,1]$, we have $-1 \leq(\mu-2 v-1) / 3 \leq S_{\lambda}(\alpha) \leq(2 \mu-v+1) / 3 \leq 1$.

Proof. From (29), we get $S_{1}(\alpha)=(2 \mu-v+1) / 3$ and $S_{0}(\alpha)=$ $(\mu-2 v-1) / 3$. Since $0 \leq \lambda \leq 1$, by Property 15 , we have $S_{0}(\alpha) \leq S_{\lambda}(\alpha) \leq S_{1}(\alpha)$. Notice that $\mu-2 v-1 \geq-3$ and $2 \mu-v+1 \leq 3$; we have

$$
\begin{aligned}
& S_{\lambda}(\alpha) \geq S_{0}(\alpha)=\frac{\mu-2 v-1}{3} \geq-1, \\
& S_{\lambda}(\alpha) \leq S_{1}(\alpha)=\frac{2 \mu-v+1}{3} \leq 1 .
\end{aligned}
$$

Therefore, Property 16 is proved.

Because the addition operation of intuitionistic fuzzy number is nonlinear, the following inequalities of preference attitudinal score function are accordingly derived.

Property 17. Let $\alpha_{1}=\left(\mu_{1}, v_{1}\right)$ and $\alpha_{2}=\left(\mu_{2}, v_{2}\right)$ be two IFNs. Denoting $\lambda_{0}=\left(1+2 v_{1}+2 v_{2}-\mu_{1} \mu_{2}-2 v_{1} v_{2}\right) /\left(\mu_{1} \mu_{2}-v_{1} v_{2}+\right.$ $\left.v_{1}+v_{2}+2\right)$, we have $\lambda_{0} \in[0,1]$, and it follows that

(1) if $\lambda=\lambda_{0}$, then $S_{\lambda}\left(\alpha_{1} \oplus \alpha_{2}\right)=S_{\lambda}\left(\alpha_{1}\right)+S_{\lambda}\left(\alpha_{2}\right)$;

(2) if $0 \leq \lambda<\lambda_{0}$, then $S_{\lambda}\left(\alpha_{1} \oplus \alpha_{2}\right)>S_{\lambda}\left(\alpha_{1}\right)+S_{\lambda}\left(\alpha_{2}\right)$;

(3) if $\lambda_{0}<\lambda \leq 1$, then $S_{\lambda}\left(\alpha_{1} \oplus \alpha_{2}\right)<S_{\lambda}\left(\alpha_{1}\right)+S_{\lambda}\left(\alpha_{2}\right)$.

Proof. Since $0 \leq \mu_{1} \mu_{2} \leq 1$ and $-1 \leq\left(v_{2}-1\right)\left(1-v_{1}\right) \leq 0$, we have

$$
\begin{aligned}
& \mu_{1} \mu_{2}-v_{1} v_{2}+v_{1}+v_{2}+2 \\
& \quad=\mu_{1} \mu_{2}+\left(v_{2}-1\right)\left(1-v_{1}\right)+3>0, \\
& 1+2 v_{1}+2 v_{2}-\mu_{1} \mu_{2}-2 v_{1} v_{2} \\
& \quad=2\left(v_{2}-1\right)\left(1-v_{1}\right)+3-\mu_{1} \mu_{2} \geq 0 .
\end{aligned}
$$

Thus, we have $\lambda_{0} \geq 0$. Considering $2 \mu_{1} \mu_{2}-\left(v_{2}-1\right)\left(1-v_{1}\right) \geq 0$ and

$$
\begin{aligned}
\mu_{1} \mu_{2} & -v_{1} v_{2}+v_{1}+v_{2}+2 \\
= & {\left[2\left(v_{2}-1\right)\left(1-v_{1}\right)+3-\mu_{1} \mu_{2}\right] } \\
& +\left[2 \mu_{1} \mu_{2}-\left(v_{2}-1\right)\left(1-v_{1}\right)\right],
\end{aligned}
$$

we have $\lambda_{0} \leq 1$.

In sum, we have $\lambda_{0} \in[0,1]$.

Denoting $f(\lambda)=S_{\lambda}\left(\alpha_{1}\right)+S_{\lambda}\left(\alpha_{2}\right)-S_{\lambda}\left(\alpha_{1} \oplus \alpha_{2}\right)$, from (28) and Definition 2, we have

$$
\begin{aligned}
f(\lambda)= & \frac{1}{3}\left(\mu_{1} \mu_{2}+2 v_{1} v_{2}-2 v_{1}-2 v_{2}-1\right) \\
& +\frac{\lambda}{3}\left(\mu_{1} \mu_{2}-v_{1} v_{2}+v_{1}+v_{2}+2\right) .
\end{aligned}
$$

If $\lambda=\lambda_{0}=\left(1+2 v_{1}+2 v_{2}-\mu_{1} \mu_{2}-2 v_{1} v_{2}\right) /\left(\mu_{1} \mu_{2}-v_{1} v_{2}+v_{1}+\right.$ $\left.v_{2}+2\right)$, then we have $f\left(\lambda_{0}\right)=0$.

Therefore, $S_{\lambda_{0}}\left(\alpha_{1} \oplus \alpha_{2}\right)=S_{\lambda_{0}}\left(\alpha_{1}\right)+S_{\lambda_{0}}\left(\alpha_{2}\right)$ holds.

Since $\mu_{1} \mu_{2}-v_{1} v_{2}+v_{1}+v_{2}+2>0$, function $f(\lambda)$ is monotonically increasing with respect to the parameter $\lambda$. Thus, if $0 \leq \lambda<\lambda_{0}$, then $f(\lambda)<f\left(\lambda_{0}\right)=0$. Based on the monotonicity of function $f(\lambda)$, we have $S_{\lambda}\left(\alpha_{1} \oplus \alpha_{2}\right)>$ $S_{\lambda}\left(\alpha_{1}\right)+S_{\lambda}\left(\alpha_{2}\right)$.

Similarly, if $\lambda_{0}<\lambda \leq 1$, then $f(\lambda)>f\left(\lambda_{0}\right)=0$; we have $S_{\lambda}\left(\alpha_{1} \oplus \alpha_{2}\right)<S_{\lambda}\left(\alpha_{1}\right)+S_{\lambda}\left(\alpha_{2}\right)$.

This completes the proof of Property 17.

Property 18. Let $\alpha=(\mu, v)$ be an IFN. $\bar{\alpha}=(v, \mu)$ is the complementary intuitionistic fuzzy number of $\alpha$. For each attitudinal parameter $\lambda \in[0,1]$, we have $S_{\lambda}(\alpha)=-S_{1-\lambda}(\bar{\alpha})$.

Proof. From (29), it follows that

$$
\begin{aligned}
S_{1-\lambda}(\bar{\alpha}) & =(1-\lambda) \frac{2 v-\mu+1}{3}+\lambda \frac{v-2 \mu-1}{3} \\
& =-\lambda \frac{2 \mu-v+1}{3}-(1-\lambda) \frac{\mu-2 v-1}{3} \\
& =-S_{\lambda}(\alpha) .
\end{aligned}
$$

Thus, Property 18 is proved.

Property 19 (sensitivity analysis). Given two IFNs $\alpha_{1}=$ $\left(\mu_{1}, v_{1}\right)$ and $\alpha_{2}=\left(\mu_{2}, v_{2}\right)$. Let $\lambda$ be the attitudinal parameter under which it has satisfied the fact that $S_{\lambda}\left(\alpha_{1}\right) \leq S_{\lambda}\left(\alpha_{2}\right)$. Let $\Delta \lambda$ be a perturbation of the attitudinal parameter $\lambda$ with $\lambda+\Delta \lambda \in[0,1]$. Then, $S_{\lambda+\Delta \lambda}\left(\alpha_{1}\right) \leq S_{\lambda+\Delta \lambda}\left(\alpha_{2}\right)$ holds if and only if

$$
\begin{aligned}
& -\lambda \leq \Delta \lambda \leq \min \left\{\frac{3 S_{\lambda}\left(\alpha_{2}\right)-3 S_{\lambda}\left(\alpha_{1}\right)}{\mu_{1}+v_{1}-\mu_{2}-v_{2}}, 1-\lambda\right\}, \\
& \mu_{1}+v_{1}>\mu_{2}+v_{2} ; \\
& \max \left\{\frac{3 S_{\lambda}\left(\alpha_{2}\right)-3 S_{\lambda}\left(\alpha_{1}\right)}{\mu_{1}+v_{1}-\mu_{2}-v_{2}},-\lambda\right\} \leq \Delta \lambda \leq 1-\lambda, \\
& \mu_{1}+v_{1}<\mu_{2}+v_{2} ; \\
& -\lambda \leq \Delta \lambda \leq 1-\lambda, \quad \mu_{1}+v_{1}=\mu_{2}+v_{2} .
\end{aligned}
$$


Proof. Since $0 \leq \lambda+\Delta \lambda \leq 1$, we have $-\lambda \leq \Delta \lambda \leq 1-\lambda$. Considering

$$
\begin{aligned}
& S_{\lambda+\Delta \lambda}\left(\alpha_{1}\right)=S_{\lambda}\left(\alpha_{1}\right)+\frac{\Delta \lambda}{3}\left(\mu_{1}+v_{1}+2\right), \\
& S_{\lambda+\Delta \lambda}\left(\alpha_{2}\right)=S_{\lambda}\left(\alpha_{2}\right)+\frac{\Delta \lambda}{3}\left(\mu_{2}+v_{2}+2\right),
\end{aligned}
$$

the following inequalities are equivalent:

$$
\begin{gathered}
S_{\lambda+\Delta \lambda}\left(\alpha_{1}\right) \leq S_{\lambda+\Delta \lambda}\left(\alpha_{2}\right) \Longleftrightarrow \\
S_{\lambda}\left(\alpha_{2}\right)-S_{\lambda}\left(\alpha_{1}\right) \geq \frac{\Delta \lambda}{3}\left(\mu_{1}+v_{1}-\mu_{2}-v_{2}\right) .
\end{gathered}
$$

We can consider (40) in three cases, shown as follows:

(a) If $\mu_{1}+v_{1}=\mu_{2}+v_{2}$, then inequalities $S_{\lambda+\Delta \lambda}\left(\alpha_{1}\right) \leq$ $S_{\lambda+\Delta \lambda}\left(\alpha_{2}\right)$ and $S_{\lambda}\left(\alpha_{2}\right)-S_{\lambda}\left(\alpha_{1}\right) \geq 0$ are equivalent.

It means that (40) is true for all $\Delta \lambda$ with $-\lambda \leq \Delta \lambda \leq 1-\lambda$.

(b) If $\mu_{1}+v_{1}>\mu_{2}+v_{2}$, then

$$
\begin{aligned}
S_{\lambda+\Delta \lambda}\left(\alpha_{1}\right) & \leq S_{\lambda+\Delta \lambda}\left(\alpha_{2}\right) \Longleftrightarrow \\
\Delta \lambda & \leq \frac{3 S_{\lambda}\left(\alpha_{2}\right)-3 S_{\lambda}\left(\alpha_{1}\right)}{\mu_{1}+v_{1}-\mu_{2}-v_{2}} .
\end{aligned}
$$

Notice that $\Delta \lambda$ is subject to the constraint $-\lambda \leq \Delta \lambda \leq 1-\lambda$, inequality $S_{\lambda+\Delta \lambda}\left(\alpha_{1}\right) \leq S_{\lambda+\Delta \lambda}\left(\alpha_{2}\right)$ is equivalent to

$$
-\lambda \leq \Delta \lambda \leq \min \left\{\frac{3 S_{\lambda}\left(\alpha_{2}\right)-3 S_{\lambda}\left(\alpha_{1}\right)}{\mu_{1}+v_{1}-\mu_{2}-v_{2}}, 1-\lambda\right\} .
$$

(c) If $\mu_{1}+v_{1}<\mu_{2}+v_{2}$, then

$$
\begin{aligned}
S_{\lambda+\Delta \lambda}\left(\alpha_{1}\right) & \leq S_{\lambda+\Delta \lambda}\left(\alpha_{2}\right) \Longleftrightarrow \\
\Delta \lambda & \geq \frac{3 S_{\lambda}\left(\alpha_{2}\right)-3 S_{\lambda}\left(\alpha_{1}\right)}{\mu_{1}+v_{1}-\mu_{2}-v_{2}} .
\end{aligned}
$$

Therefore, inequality $S_{\lambda+\Delta \lambda}\left(\alpha_{1}\right) \leq S_{\lambda+\Delta \lambda}\left(\alpha_{2}\right)$ is equivalent to

$$
\max \left\{\frac{3 S_{\lambda}\left(\alpha_{2}\right)-3 S_{\lambda}\left(\alpha_{1}\right)}{\mu_{1}+v_{1}-\mu_{2}-v_{2}},-\lambda\right\} \leq \Delta \lambda \leq 1-\lambda
$$

In sum, Property 19 is proved.

3.3. The Relations among Score Functions and Accuracy Functions. In the following, the relations among score functions and accuracy functions are studied in detail.
Property 20. Let $\alpha=(\mu, v)$ be an IFN. The score function $S_{X}(\alpha)$, accuracy function $A_{X}(\alpha)$, and preference attitudinal score function $S_{\lambda}(\alpha)$ are related as

$$
S_{\lambda}(\alpha)=\frac{1}{2} S_{X}(\alpha)+\frac{2 \lambda-1}{6} A_{X}(\alpha)+\frac{2 \lambda-1}{3} .
$$

In particular, $S_{0.5}(\alpha)=(1 / 2) S_{X}(\alpha)$ holds.

Proof. The proof is straightforward, so omitted.

Property 21. Let $\alpha=(\mu, v)$ be an IFN. The score function $S_{X}(\alpha)$, accuracy function $A_{X}(\alpha)$, and preference attitudinal accuracy function $A_{\lambda}(\alpha)$ are related as

$$
A_{\lambda}(\alpha)=\frac{1}{6} A_{X}(\alpha)+\frac{2 \lambda-1}{2} S_{X}(\alpha)+\frac{1}{3} .
$$

In particular, $A_{0.5}(\alpha)=(1 / 6) A_{X}(\alpha)+1 / 3$ holds.

Proof. The proof is straightforward, so omitted.

Property 22. Let $\alpha=(\mu, v)$ be an IFN. The preference attitudinal score function $S_{\lambda}(\alpha)$ and accuracy function $A_{\lambda}(\alpha)$ are related as

$$
\begin{gathered}
(\mu+v+2) A_{\lambda}(\alpha)+(3 v-3 \mu) S_{\lambda}(\alpha) \\
=\frac{2}{3}(2 \mu-v+1)(2 v-\mu+1) .
\end{gathered}
$$

Proof. From (8) and (28), we have

$$
\begin{aligned}
(\mu+ & v+2) A_{\lambda}(\alpha)+(3 v-3 \mu) S_{X}(\alpha) \\
= & (\mu+v+2)\left(\frac{1-\mu+2 v}{3}+\lambda(\mu-v)\right) \\
& +(3 v-3 \mu)\left(\frac{\mu-2 v-1}{3}+\frac{\lambda}{3}(\mu+v+2)\right) \\
= & \frac{1}{3}(\mu+v+2)(1-\mu+2 v) \\
& +\frac{1}{3}(3 v-3 \mu)(\mu-2 v-1) \\
= & \frac{2}{3}(2 \mu-v+1)(2 v-\mu+1) .
\end{aligned}
$$

Thus, Property 22 is proved.

Property 23. Let $\alpha=(\mu, v)$ be an IFN. Given two attitudinal parameters $\lambda_{1}$ and $\lambda_{2}$, such that $\lambda_{1}<\lambda_{2}$. The score function $S_{X}(\alpha)$ and preference attitudinal accuracy function $A_{\lambda}(\alpha)$ are related as follows:

$$
\begin{aligned}
& \text { (1) If } S_{X}(\alpha)>0 \text {, then } A_{\lambda_{1}}(\alpha)<A_{\lambda_{2}}(\alpha) \text {. } \\
& \text { (2) If } S_{X}(\alpha)<0 \text {, then } A_{\lambda_{1}}(\alpha)>A_{\lambda_{2}}(\alpha) \text {. } \\
& \text { (3) If } S_{X}(\alpha)=0 \text {, then } A_{\lambda_{1}}(\alpha)=A_{\lambda_{2}}(\alpha) \text {. }
\end{aligned}
$$

Proof. From (8), we have

$$
\begin{aligned}
& A_{\lambda_{1}}(\alpha)=\frac{1-\mu+2 v}{3}+\lambda_{1}(\mu-v), \\
& A_{\lambda_{2}}(\alpha)=\frac{1-\mu+2 v}{3}+\lambda_{2}(\mu-v) .
\end{aligned}
$$


Thus, we have $A_{\lambda_{1}}(\alpha)-A_{\lambda_{2}}(\alpha)=\left(\lambda_{1}-\lambda_{2}\right)(\mu-v)$. Since $\lambda_{1}<\lambda_{2}$ and $S_{X}(\alpha)=\mu-v$, items (1), (2), and (3) are obviously hold.

Therefore, Property 23 is proved.

Property 24. Let $\alpha=(\mu, v)$ be an IFN. For all $\lambda \in[0,1]$, $A_{\lambda}(\alpha) \geq S_{\lambda}(\alpha)$ holds. In particular, we have $A_{1}(\alpha)=S_{1}(\alpha)$, $A_{0}(\alpha)=-S_{0}(\alpha), A_{\lambda}((1,0))=S_{\lambda}((1,0))$, and $A_{\lambda}((0,1))=$ $-S_{\lambda}((0,1))$.

Proof. From (8) and (28), we have

$$
A_{\lambda}(\alpha)-S_{\lambda}(\alpha)=\frac{1}{3}(2+4 v-2 \mu)(1-\lambda) .
$$

Since $2+4 v-2 \mu \geq 0$ and $\lambda \in[0,1]$, we have $A_{\lambda}(\alpha)-S_{\lambda}(\alpha) \geq 0$. Obviously, $A_{1}(\alpha)=S_{1}(\alpha)=(2 \mu-v+1) / 3, A_{0}(\alpha)=$ $-S_{0}(\alpha)=(2 v-\mu+1) / 3$.

When $\alpha=(1,0)$, we have $A_{\lambda}(\alpha)=S_{\lambda}(\alpha)=\lambda$. When $\alpha=(0,1)$, we have $A_{\lambda}(\alpha)=-S_{\lambda}(\alpha)=1-\lambda$.

This completes the proof of Property 24 .

\section{The Order Relation between Intuitionistic Fuzzy Numbers}

In this section, a total order is established based on the proposed score and accuracy functions.

Property 25. Let $\alpha_{1}=\left(\mu_{1}, v_{1}\right)$ and $\alpha_{2}=\left(\mu_{2}, v_{2}\right)$ be two IFNs. For each $\lambda \in[0,1]$, we can get the equivalence as follows:

$$
\begin{aligned}
\alpha_{1} & =\alpha_{2} \Longleftrightarrow \\
A_{\lambda}\left(\alpha_{1}\right) & =A_{\lambda}\left(\alpha_{2}\right), \\
S_{\lambda}\left(\alpha_{1}\right) & =S_{\lambda}\left(\alpha_{2}\right), \\
\pi\left(\alpha_{1}\right) & =\pi\left(\alpha_{2}\right) .
\end{aligned}
$$

Proof. " $\Rightarrow$ " If $\alpha_{1}=\alpha_{2}$, we have $\mu_{1}=\mu_{2}$ and $v_{1}=v_{2}$. Obviously, $A_{\lambda}\left(\alpha_{1}\right)=A_{\lambda}\left(\alpha_{2}\right), S_{\lambda}\left(\alpha_{1}\right)=S_{\lambda}\left(\alpha_{2}\right)$, and $\pi\left(\alpha_{1}\right)=$ $\pi\left(\alpha_{2}\right)$ holds.

" $\Leftarrow$ " In the following, the proof of sufficiency is considered in three cases.

(1) When $\lambda \in(0,1)$, since $A_{\lambda}\left(\alpha_{1}\right)=A_{\lambda}\left(\alpha_{2}\right)$ and $S_{\lambda}\left(\alpha_{1}\right)=$ $S_{\lambda}\left(\alpha_{2}\right)$, we have

$$
\begin{aligned}
& \frac{1-\mu_{1}+2 v_{1}}{3}+\lambda\left(\mu_{1}-v_{1}\right) \\
& =\frac{1-\mu_{2}+2 v_{2}}{3}+\lambda\left(\mu_{2}-v_{2}\right), \\
& \frac{\mu_{1}-2 v_{1}-1}{3}+\frac{\lambda}{3}\left(\mu_{1}+v_{1}+2\right) \\
& =\frac{\mu_{2}-2 v_{2}-1}{3}+\frac{\lambda}{3}\left(\mu_{2}+v_{2}+2\right) .
\end{aligned}
$$

Equation (52) can be further written as

$$
\begin{gathered}
(3 \lambda-1)\left(\mu_{1}-\mu_{2}\right)=(3 \lambda-2)\left(v_{1}-v_{2}\right), \\
(\lambda+1)\left(\mu_{1}-\mu_{2}\right)=(2-\lambda)\left(v_{1}-v_{2}\right) .
\end{gathered}
$$

From (54), we have

$$
\mu_{1}-\mu_{2}=\frac{2-\lambda}{\lambda+1}\left(v_{1}-v_{2}\right)
$$

Applying (55) to (53), we have

$$
\frac{(2-\lambda)(3 \lambda-1)}{\lambda+1}\left(v_{1}-v_{2}\right)=(3 \lambda-2)\left(v_{1}-v_{2}\right)
$$

which can be equivalently expressed by $\lambda(1-\lambda)\left(v_{1}-v_{2}\right)=0$.

Considering $\lambda \in(0,1)$, we get $v_{1}=v_{2}$. From (54) we have $\mu_{1}=\mu_{2}$.

(2) When $\lambda=0$, since $A_{0}\left(\alpha_{1}\right)=A_{0}\left(\alpha_{2}\right), S_{0}\left(\alpha_{1}\right)=S_{0}\left(\alpha_{2}\right)$, and $\pi\left(\alpha_{1}\right)=\pi\left(\alpha_{2}\right)$, we have $\mu_{1}-\mu_{2}=2\left(v_{1}-v_{2}\right)$ and $\mu_{1}+v_{1}=$ $\mu_{2}+v_{2}$. Thus, we get $\mu_{1}=\mu_{2}$ and $v_{1}=v_{2}$.

(3) When $\lambda=1$, since $A_{1}\left(\alpha_{1}\right)=A_{1}\left(\alpha_{2}\right), S_{1}\left(\alpha_{1}\right)=S_{1}\left(\alpha_{2}\right)$, and $\pi\left(\alpha_{1}\right)=\pi\left(\alpha_{2}\right)$, we have $v_{1}-v_{2}=2\left(\mu_{1}-\mu_{2}\right)$ and $\mu_{1}+v_{1}=$ $\mu_{2}+v_{2}$. Therefore, we also get $\mu_{1}=\mu_{2}$ and $v_{1}=v_{2}$.

In sum, for each $\lambda \in[0,1]$, we have $\mu_{1}=\mu_{2}$ and $v_{1}=v_{2}$. Namely, $\alpha_{1}=\alpha_{2}$ holds.

The order relation on the set of intuitionistic fuzzy numbers can be defined as follows.

Definition 26. Let $\alpha_{1}=\left(\mu_{1}, v_{1}\right)$ and $\alpha_{2}=\left(\mu_{2}, v_{2}\right)$ be two IFNs. $\forall \lambda \in[0,1], \alpha_{1}$ is said to be inferior to $\alpha_{2}$, denoted by $\alpha_{1} \prec_{\lambda} \alpha_{2}$, if and only if one of the following conditions is true:

(1) $S_{\lambda}\left(\alpha_{1}\right)<S_{\lambda}\left(\alpha_{2}\right)$.

(2) $S_{\lambda}\left(\alpha_{1}\right)=S_{\lambda}\left(\alpha_{2}\right)$ and $A_{\lambda}\left(\alpha_{1}\right)<A_{\lambda}\left(\alpha_{2}\right)$.

(3) $S_{\lambda}\left(\alpha_{1}\right)=S_{\lambda}\left(\alpha_{2}\right), A_{\lambda}\left(\alpha_{1}\right)=A_{\lambda}\left(\alpha_{2}\right)$, and $\pi\left(\alpha_{1}\right)>$ $\pi\left(\alpha_{2}\right)$.

Theorem 27. The relation $\prec_{\lambda}$ on the set of IFNs is a strict order, that is, the relation $\prec_{\lambda}$ satisfying the following:

(1) Irreflexivity: $\forall \alpha \in I N F, \alpha \prec_{\lambda} \alpha$ does not hold.

(2) Asymmetry: $\forall \alpha_{1}, \alpha_{2} \in I N F$, if $\alpha_{1} \prec_{\lambda} \alpha_{2}$, then $\alpha_{2} \prec_{\lambda} \alpha_{1}$ does not hold.

(3) Transitivity: $\forall \alpha_{1}, \alpha_{2}, \alpha_{3} \in I N F$, if $\alpha_{1} \prec_{\lambda} \alpha_{2}$ and $\alpha_{2} \prec_{\lambda} \alpha_{3}$, then $\alpha_{1} \prec_{\lambda} \alpha_{3}$.

Proof. According to Definition 26, Properties (1) and (2) obviously hold here. In the following, we focus on the proof of Property (3).

Since $\alpha_{1} \prec_{\lambda} \alpha_{2}$, from Definition 26, the proof of transitivity property is considered in three cases.

(a) $S_{\lambda}\left(\alpha_{1}\right)<S_{\lambda}\left(\alpha_{2}\right)$. Because $\alpha_{2} \prec_{\lambda} \alpha_{3}$, we have $S_{\lambda}\left(\alpha_{2}\right) \leq$ $S_{\lambda}\left(\alpha_{3}\right)$. Thus, $S_{\lambda}\left(\alpha_{1}\right)<S_{\lambda}\left(\alpha_{3}\right)$ holds. Namely, from Definition 26, we have $\alpha_{1} \prec_{\lambda} \alpha_{3}$. 
(b) $S_{\lambda}\left(\alpha_{1}\right)=S_{\lambda}\left(\alpha_{2}\right)$ and $A_{\lambda}\left(\alpha_{1}\right)<A_{\lambda}\left(\alpha_{2}\right)$. Because $\alpha_{2} \prec_{\lambda} \alpha_{3}$, one has the following:

(b1) If $S_{\lambda}\left(\alpha_{2}\right)<S_{\lambda}\left(\alpha_{3}\right)$, then $S_{\lambda}\left(\alpha_{1}\right)=S_{\lambda}\left(\alpha_{2}\right)<$ $S_{\lambda}\left(\alpha_{3}\right)$. We conclude that $\alpha_{1} \prec_{\lambda} \alpha_{3}$.

(b2) If $S_{\lambda}\left(\alpha_{2}\right)=S_{\lambda}\left(\alpha_{3}\right)$ and $A_{\lambda}\left(\alpha_{2}\right)<A_{\lambda}\left(\alpha_{3}\right)$, then $S_{\lambda}\left(\alpha_{1}\right)=S_{\lambda}\left(\alpha_{3}\right)$ and $A_{\lambda}\left(\alpha_{1}\right)<A_{\lambda}\left(\alpha_{2}\right)<$ $A_{\lambda}\left(\alpha_{3}\right)$. We have $\alpha_{1}<_{\lambda} \alpha_{3}$.

(b3) If $S_{\lambda}\left(\alpha_{2}\right)=S_{\lambda}\left(\alpha_{3}\right), A_{\lambda}\left(\alpha_{2}\right)=A_{\lambda}\left(\alpha_{3}\right)$, and $\pi\left(\alpha_{2}\right)>\pi\left(\alpha_{3}\right)$, then $S_{\lambda}\left(\alpha_{1}\right)=S_{\lambda}\left(\alpha_{3}\right), A_{\lambda}\left(\alpha_{1}\right)<$ $A_{\lambda}\left(\alpha_{2}\right)=A_{\lambda}\left(\alpha_{3}\right)$. We get $\alpha_{1} \prec_{\lambda} \alpha_{3}$.

(c) $S_{\lambda}\left(\alpha_{1}\right)=S_{\lambda}\left(\alpha_{2}\right), A_{\lambda}\left(\alpha_{1}\right)=A_{\lambda}\left(\alpha_{2}\right)$ and $\pi\left(\alpha_{1}\right)>\pi\left(\alpha_{2}\right)$. Because $\alpha_{2} \prec_{\lambda} \alpha_{3}$, then

(c1) if $S_{\lambda}\left(\alpha_{2}\right)<S_{\lambda}\left(\alpha_{3}\right)$, then $S_{\lambda}\left(\alpha_{1}\right)=S_{\lambda}\left(\alpha_{2}\right)<$ $S_{\lambda}\left(\alpha_{3}\right)$. We conclude that $\alpha_{1} \prec_{\lambda} \alpha_{3}$.

(c2) if $S_{\lambda}\left(\alpha_{2}\right)=S_{\lambda}\left(\alpha_{3}\right)$ and $A_{\lambda}\left(\alpha_{2}\right)<A_{\lambda}\left(\alpha_{3}\right)$, then $S_{\lambda}\left(\alpha_{1}\right)=S_{\lambda}\left(\alpha_{3}\right)$ and $A_{\lambda}\left(\alpha_{1}\right)=A_{\lambda}\left(\alpha_{2}\right)<$ $A_{\lambda}\left(\alpha_{3}\right)$. We have $\alpha_{1}<_{\lambda} \alpha_{3}$.

(c3) if $S_{\lambda}\left(\alpha_{2}\right)=S_{\lambda}\left(\alpha_{3}\right), A_{\lambda}\left(\alpha_{2}\right)=A_{\lambda}\left(\alpha_{3}\right)$, and $\pi\left(\alpha_{2}\right)>\pi\left(\alpha_{3}\right)$, then $S_{\lambda}\left(\alpha_{1}\right)=S_{\lambda}\left(\alpha_{3}\right), A_{\lambda}\left(\alpha_{1}\right)=$ $A_{\lambda}\left(\alpha_{3}\right)$, and $\pi\left(\alpha_{1}\right)>\pi\left(\alpha_{2}\right)>\pi\left(\alpha_{3}\right)$. We get $\alpha_{1} \prec_{\lambda} \alpha_{3}$.

In sum, if $\alpha_{1} \prec_{\lambda} \alpha_{2}$ and $\alpha_{2} \prec_{\lambda} \alpha_{3}$, then $\alpha_{1} \prec_{\lambda} \alpha_{3}$ always hold here. Therefore, Theorem 27 is proved.

Property 28. Let $\alpha_{1}=\left(\mu_{1}, v_{1}\right)$ and $\alpha_{2}=\left(\mu_{2}, v_{2}\right)$ be two IFNs. $\forall \lambda \in[0,1]$, if $S_{X}\left(\alpha_{1}\right)=S_{X}\left(\alpha_{2}\right)$ and $A_{X}\left(\alpha_{1}\right)<A_{X}\left(\alpha_{2}\right)$ (which imply that $\alpha_{1} \prec_{X} \alpha_{2}$ ), then one has the following:

(1) If $\lambda>1 / 2$, then $S_{\lambda}\left(\alpha_{1}\right)<S_{\lambda}\left(\alpha_{2}\right)$; we get $\alpha_{1} \prec_{\lambda} \alpha_{2}$.

(2) If $\lambda=1 / 2$, then $S_{\lambda}\left(\alpha_{2}\right)=S_{\lambda}\left(\alpha_{1}\right)$ and $A_{\lambda}\left(\alpha_{1}\right)<$ $A_{\lambda}\left(\alpha_{2}\right)$; we get $\alpha_{1} \prec_{\lambda} \alpha_{2}$.

(3) If $\lambda<1 / 2$, then $S_{\lambda}\left(\alpha_{2}\right)<S_{\lambda}\left(\alpha_{1}\right)$; we get $\alpha_{2} \prec_{\lambda} \alpha_{1}$.

Proof. Since $S_{X}\left(\alpha_{1}\right)=S_{X}\left(\alpha_{2}\right)$ and $A_{X}\left(\alpha_{1}\right)<A_{X}\left(\alpha_{2}\right)$, we have $\mu_{1}-v_{1}=\mu_{2}-v_{2}$ and $\mu_{1}+v_{1}<\mu_{2}+v_{2}$. Based on (28), we have

$$
\begin{aligned}
S_{\lambda}\left(\alpha_{1}\right)-S_{\lambda}\left(\alpha_{2}\right)= & \frac{1}{3}\left(\mu_{1}-2 v_{1}-\mu_{2}+2 v_{2}\right) \\
& +\frac{\lambda}{3}\left(\mu_{1}+v_{1}-\mu_{2}-v_{2}\right) .
\end{aligned}
$$
have

(1) If $\lambda>1 / 2$, by considering $\mu_{1}+v_{1}-\mu_{2}-v_{2}<0$, we

$$
S_{\lambda}\left(\alpha_{1}\right)-S_{\lambda}\left(\alpha_{2}\right)<\frac{1}{2}\left(\mu_{1}-\mu_{2}+v_{2}-v_{1}\right)=0 .
$$

Namely, $S_{\lambda}\left(\alpha_{1}\right)<S_{\lambda}\left(\alpha_{2}\right)$ holds. Thus, we have $\alpha_{1} \prec_{\lambda} \alpha_{2}$.

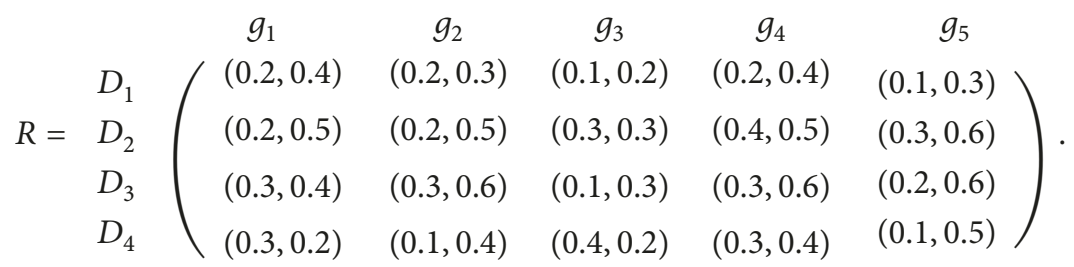

(2) If $\lambda=1 / 2$, then $S_{0.5}\left(\alpha_{1}\right)-S_{0.5}\left(\alpha_{2}\right)=(1 / 2)\left(\mu_{1}-\mu_{2}+\right.$ $\left.v_{2}-v_{1}\right)=0$.

Namely, $S_{0.5}\left(\alpha_{1}\right)=S_{0.5}\left(\alpha_{2}\right)$ holds.

Moreover, from (8), we get $A_{0.5}\left(\alpha_{1}\right)-A_{0.5}\left(\alpha_{2}\right)=$ $(1 / 6)\left(\mu_{1}+v_{1}-\mu_{2}-v_{2}\right)<0$

Therefore, we have $\alpha_{1} \prec_{\lambda} \alpha_{2}$.

(3) If $\lambda<1 / 2$, by considering $\mu_{1}+v_{1}-\mu_{2}-v_{2}<0$, we have

$$
S_{\lambda}\left(\alpha_{1}\right)-S_{\lambda}\left(\alpha_{2}\right)>\frac{1}{2}\left(\mu_{1}-\mu_{2}+v_{2}-v_{1}\right)=0 .
$$

Namely, $S_{\lambda}\left(\alpha_{2}\right)<S_{\lambda}\left(\alpha_{1}\right)$ holds. Thus, we have $\alpha_{2} \prec_{\lambda} \alpha_{1}$.

In sum, Property 28 is proved.

Remark 29. When $\lambda=0.5$, from Properties 20 and 21, we have $S_{0.5}(\alpha)=(1 / 2) S_{X}(\alpha)$ and $A_{0.5}(\alpha)=(1 / 6) A_{X}(\alpha)+1 / 3$. In this case, the order relation proposed in Definition 26 is equivalent to Xu's method in Definition 4. That is to say, Xu's method is a special case of the proposed method.

\section{Numerical Examples and Comparison}

In this section, the proposed ranking method is applied to select renewable energy. Moreover, some comparison examples are employed to show the advantage and validity of the proposed method.

5.1. Application of the Preference Attitudinal Score and Accuracy Functions in Renewable Energy Selection. Due to the increasing consumption of fossil fuels, the shortage of energy resources and the pollution caused by burning energy become more and more serious. It is necessary to relieve energy shortage and environmental pollution by developing renewable energy sources in the future. To find efficient alternative energy sources, many governments around the world focus on the renewable energy selection problems in the past several decades (adapted from [36]). Suppose there is a panel with four renewable energy resources $D_{1}$ (wind energy), $D_{2}$ (hydropower), $D_{3}$ (geothermal), and $D_{4}$ (solar power) to be chose. Government wants to select a renewable energy in the best option according to the following five attributes: $g_{1}$ (environmental), $g_{2}$ (sociopolitical), $g_{3}$ (quality of the energy source), $g_{4}$ (technological), and $g_{5}$ (economic). The weighting vector of attributes is $W=\left(w_{1}, w_{2}, w_{3}, w_{4}, w_{5}\right)=$ $(0.2,0.1,0.2,0.4,0.1)$. Since intuitionistic fuzzy set is practical and flexible in dealing with uncertainty and ambiguity, the four possible renewable energy resources $D_{1}, D_{2}, D_{3}$, and $D_{4}$ are evaluated using intuitionistic fuzzy numbers under five attributes. Then, the intuitionistic fuzzy decision matrix $R=\left(r_{i j}\right)_{4 \times 5}$ is constructed as follows:

$\left.\begin{array}{ccc}g_{3} & g_{4} & g_{5} \\ (0.1,0.2) & (0.2,0.4) & (0.1,0.3) \\ (0.3,0.3) & (0.4,0.5) & (0.3,0.6) \\ (0.1,0.3) & (0.3,0.6) & (0.2,0.6) \\ (0.4,0.2) & (0.3,0.4) & (0.1,0.5)\end{array}\right)$


Based on (28), the preference attitudinal score matrix $S_{\lambda}(R)=\left(S_{\lambda}\left(r_{i j}\right)\right)_{4 \times 5}$ corresponding to attitudinal parameter $\lambda$ is calculated as

$$
S_{\lambda}(R)=\frac{1}{3} \cdot\left(\begin{array}{rrrrr}
-1.6+2.6 \lambda & -1.4+2.5 \lambda & -1.3+2.3 \lambda & -1.6+2.6 \lambda & -1.5+2.4 \lambda \\
-1.8+2.7 \lambda & -1.8+2.7 \lambda & -1.3+2.6 \lambda & -1.6+2.9 \lambda & -1.9+2.9 \lambda \\
-1.5+2.7 \lambda & -1.9+2.9 \lambda & -1.5+2.4 \lambda & -1.9+2.9 \lambda & -2.0+2.8 \lambda \\
-1.1+2.5 \lambda & -1.7+2.5 \lambda & -1.0+2.6 \lambda & -1.5+2.7 \lambda & -1.9+2.6 \lambda
\end{array}\right) \text {. }
$$

In the following, we discuss the order relation of alternatives in three cases.

(1) The collective preference attitudinal score values of alternatives $D_{1}, D_{2}, D_{3}$, and $D_{4}$ are derived as follows:

$$
\begin{aligned}
& S_{\lambda}\left(D_{1}\right)=\sum_{j=1}^{5} w_{j} S_{\lambda}\left(r_{1 j}\right)=\frac{1}{3}(-1.51+2.51 \lambda) ; \\
& S_{\lambda}\left(D_{2}\right)=\sum_{j=1}^{5} w_{j} S_{\lambda}\left(r_{2 j}\right)=\frac{1}{3}(-1.63+2.78 \lambda) ; \\
& S_{\lambda}\left(D_{3}\right)=\sum_{j=1}^{5} w_{j} S_{\lambda}\left(r_{3 j}\right)=\frac{1}{3}(-1.75+2.75 \lambda) ; \\
& S_{\lambda}\left(D_{4}\right)=\sum_{j=1}^{5} w_{j} S_{\lambda}\left(r_{4 j}\right)=\frac{1}{3}(-1.38+2.61 \lambda) .
\end{aligned}
$$

It follows that

$$
\begin{aligned}
& S_{\lambda}\left(D_{4}\right)-S_{\lambda}\left(D_{1}\right)=\frac{1}{3}(0.13+0.10 \lambda)>0 ; \\
& S_{\lambda}\left(D_{4}\right)-S_{\lambda}\left(D_{2}\right)=\frac{1}{3}(0.25-0.17 \lambda)>0 ; \\
& S_{\lambda}\left(D_{4}\right)-S_{\lambda}\left(D_{3}\right)=\frac{1}{3}(0.37-0.14 \lambda)>0 ; \\
& S_{\lambda}\left(D_{3}\right)-S_{\lambda}\left(D_{2}\right)=\frac{1}{3}(-0.12-0.03 \lambda)<0 .
\end{aligned}
$$

Accordingly, for all $\lambda \in[0,1]$, we have $S_{\lambda}\left(D_{4}\right)>S_{\lambda}\left(D_{2}\right)>$ $S_{\lambda}\left(D_{3}\right)$ and $S_{\lambda}\left(D_{4}\right)>S_{\lambda}\left(D_{1}\right)$. From Definition 26, we have $D_{4}>D_{2}>D_{3}$ and $D_{4}>D_{1}$.

(2) Moreover, we have $S_{\lambda}\left(D_{1}\right)-S_{\lambda}\left(D_{2}\right)=(1 / 3)(0.12-$ $0.27 \lambda)$.

If $\lambda \in[0,4 / 9)$, then we have $S_{\lambda}\left(D_{1}\right)>S_{\lambda}\left(D_{2}\right)$. Namely, $D_{1}>D_{2}$.

If $\lambda \in(4 / 9,1]$, then we have $S_{\lambda}\left(D_{2}\right)>S_{\lambda}\left(D_{1}\right)$. Namely, $D_{2}>D_{1}$.

If $\lambda=4 / 9$, then we have $S_{\lambda}\left(D_{2}\right)=S_{\lambda}\left(D_{1}\right)$. In this case, we need to consider the preference attitudinal accuracy values as follows:

$$
\begin{aligned}
& A_{4 / 9}\left(r_{11}\right)=0.44, \\
& A_{4 / 9}\left(r_{12}\right)=0.42,
\end{aligned}
$$

$$
\begin{aligned}
& A_{4 / 9}\left(r_{13}\right)=0.39, \\
& A_{4 / 9}\left(r_{14}\right)=0.44, \\
& A_{4 / 9}\left(r_{15}\right)=0.41 ; \\
& A_{4 / 9}\left(r_{21}\right)=0.47, \\
& A_{4 / 9}\left(r_{22}\right)=0.47, \\
& A_{4 / 9}\left(r_{23}\right)=0.43, \\
& A_{4 / 9}\left(r_{24}\right)=0.49, \\
& A_{4 / 9}\left(r_{25}\right)=0.50 .
\end{aligned}
$$

Therefore, the collective preference attitudinal accuracy values of alternatives $D_{1}$ and $D_{2}$ are derived as

$$
\begin{aligned}
& A_{4 / 9}\left(D_{1}\right)=\sum_{j=1}^{5} w_{j} A_{4 / 9}\left(r_{1 j}\right)=0.425, \\
& A_{4 / 9}\left(D_{2}\right)=\sum_{j=1}^{5} w_{j} A_{4 / 9}\left(r_{2 j}\right)=0.473 .
\end{aligned}
$$

It follows that $A_{4 / 9}\left(D_{2}\right)>A_{4 / 9}\left(D_{1}\right)$. From Definition 26, we have $D_{2}>D_{1}$.

(3) In addition, $S_{\lambda}\left(D_{1}\right)-S_{\lambda}\left(D_{3}\right)=(1 / 3)(0.24-0.24 \lambda)$. Obviously, $\forall \lambda \in[0,1)$ we have $S_{\lambda}\left(D_{1}\right)>S_{\lambda}\left(D_{3}\right)$ : that is, $D_{1}>$ $D_{3}$.

We observe that $S_{\lambda}\left(D_{1}\right)=S_{\lambda}\left(D_{3}\right)$ if and only if $\lambda=1$. In this case, we cannot decide the best one between $D_{1}$ and $\mathrm{D}_{3}$ based on collective preference attitudinal score values. To select the best one, we consider the preference attitudinal accuracy values as follows:

$$
\begin{aligned}
& A_{1}\left(r_{11}\right)=\frac{1}{3}, \\
& A_{1}\left(r_{12}\right)=\frac{11}{30}, \\
& A_{1}\left(r_{13}\right)=\frac{1}{3}, \\
& A_{1}\left(r_{14}\right)=\frac{1}{3},
\end{aligned}
$$




$$
\begin{aligned}
& A_{1}\left(r_{15}\right)=\frac{3}{10} ; \\
& A_{1}\left(r_{31}\right)=\frac{2}{5}, \\
& A_{1}\left(r_{32}\right)=\frac{1}{3}, \\
& A_{1}\left(r_{33}\right)=\frac{3}{10}, \\
& A_{1}\left(r_{34}\right)=\frac{1}{3}, \\
& A_{1}\left(r_{35}\right)=\frac{4}{15} .
\end{aligned}
$$

Thus, the collective preference attitudinal accuracy values of alternatives $D_{1}$ and $D_{3}$ are derived as $A_{1}\left(D_{1}\right)=$ $\sum_{j=1}^{5} w_{j} A_{1}\left(r_{1 j}\right)=1 / 3$ and $A_{1}\left(D_{3}\right)=\sum_{j=1}^{5} w_{j} A_{1}\left(r_{3 j}\right)=1 / 3$.

From Definition 26, we need further to consider the hesitation degree as follows:

$$
\begin{aligned}
& \pi\left(r_{11}\right)=0.4, \\
& \pi\left(r_{12}\right)=0.5, \\
& \pi\left(r_{13}\right)=0.7 \\
& \pi\left(r_{14}\right)=0.4 \\
& \pi\left(r_{15}\right)=0.6 \\
& \pi\left(r_{31}\right)=0.3, \\
& \pi\left(r_{32}\right)=0.1, \\
& \pi\left(r_{33}\right)=0.6, \\
& \pi\left(r_{34}\right)=0.1, \\
& \pi\left(r_{35}\right)=0.2 .
\end{aligned}
$$

Accordingly, the collective hesitation degree of alternatives $D_{1}$ and $D_{3}$ is calculated as

$$
\begin{aligned}
& \pi\left(D_{1}\right)=\sum_{j=1}^{5} w_{j} \pi\left(r_{1 j}\right)=0.49, \\
& \pi\left(D_{3}\right)=\sum_{j=1}^{5} w_{j} \pi\left(r_{3 j}\right)=0.25 .
\end{aligned}
$$

It follows that $\pi\left(D_{1}\right)>\pi\left(D_{3}\right)$. From Definition 26, we have $D_{3}>D_{1}$. tives:

In sum, we have the following order relations of alterna-
(a) If $\lambda \in[0,4 / 9)$, then $D_{4}>D_{1}>D_{2}>D_{3}$.
(b) If $\lambda \in[4 / 9,1)$, then $D_{4}>D_{2}>D_{1}>D_{3}$.
(c) If $\lambda=1$, then $D_{4}>D_{2}>D_{3}>D_{1}$.

5.2. Comparison with Other Works. Suppose that we need to rank four intuitionistic fuzzy numbers as follows:

$$
\begin{aligned}
& \alpha_{1}=(0.4,0.2), \\
& \alpha_{2}=(0.5,0.3), \\
& \alpha_{3}=(0.3,0.4), \\
& \alpha_{4}=(0.4,0.5) .
\end{aligned}
$$

By (28), the preference attitudinal score values of $\alpha_{1}, \alpha_{2}, \alpha_{3}$, and $\alpha_{4}$ are calculated as

$$
\begin{aligned}
& S_{\lambda}\left(\alpha_{1}\right)=\frac{1}{3}(-1+2.6 \lambda), \\
& S_{\lambda}\left(\alpha_{2}\right)=\frac{1}{3}(-1.1+2.8 \lambda), \\
& S_{\lambda}\left(\alpha_{3}\right)=\frac{1}{3}(-1.5+2.7 \lambda), \\
& S_{\lambda}\left(\alpha_{4}\right)=\frac{1}{3}(-1.6+2.9 \lambda) .
\end{aligned}
$$

Since $\lambda \in[0,1]$, it follows that

$$
\begin{aligned}
& S_{\lambda}\left(\alpha_{3}\right)-S_{\lambda}\left(\alpha_{1}\right)=-0.5+0.1 \lambda<0, \\
& S_{\lambda}\left(\alpha_{4}\right)-S_{\lambda}\left(\alpha_{1}\right)=-0.6+0.3 \lambda<0, \\
& S_{\lambda}\left(\alpha_{3}\right)-S_{\lambda}\left(\alpha_{2}\right)=-0.4-0.1 \lambda<0, \\
& S_{\lambda}\left(\alpha_{4}\right)-S_{\lambda}\left(\alpha_{2}\right)=-0.5+0.1 \lambda<0 .
\end{aligned}
$$

Clearly, we have $S_{\lambda}\left(\alpha_{3}\right)<S_{\lambda}\left(\alpha_{1}\right), S_{\lambda}\left(\alpha_{4}\right)<S_{\lambda}\left(\alpha_{1}\right), S_{\lambda}\left(\alpha_{3}\right)<$ $S_{\lambda}\left(\alpha_{2}\right)$, and $S_{\lambda}\left(\alpha_{4}\right)<S_{\lambda}\left(\alpha_{2}\right)$. Based on Definition 26, we have $\alpha_{3} \prec_{\lambda} \alpha_{1}, \alpha_{4} \prec_{\lambda} \alpha_{1}, \alpha_{3} \prec_{\lambda} \alpha_{2}$, and $\alpha_{4} \prec_{\lambda} \alpha_{2}$.

Moreover, we have $S_{\lambda}\left(\alpha_{2}\right)-S_{\lambda}\left(\alpha_{1}\right)=-0.1+0.2 \lambda$ and $S_{\lambda}\left(\alpha_{4}\right)-S_{\lambda}\left(\alpha_{3}\right)=-0.1+0.2 \lambda$. If $\lambda \in[0,0.5)$, then $S_{\lambda}\left(\alpha_{2}\right)<S_{\lambda}\left(\alpha_{1}\right)$ and $S_{\lambda}\left(\alpha_{4}\right)<S_{\lambda}\left(\alpha_{3}\right)$; we have $\alpha_{2} \prec_{\lambda} \alpha_{1}$ and $\alpha_{4} \prec_{\lambda} \alpha_{3}$. If $\lambda \in(0.5,1]$, then $S_{\lambda}\left(\alpha_{2}\right)>S_{\lambda}\left(\alpha_{1}\right)$ and $S_{\lambda}\left(\alpha_{4}\right)>$ $S_{\lambda}\left(\alpha_{3}\right)$; we have $\alpha_{1} \prec_{\lambda} \alpha_{2}$ and $\alpha_{3} \prec_{\lambda} \alpha_{4}$. We observe that $S_{0.5}\left(\alpha_{1}\right)=S_{0.5}\left(\alpha_{2}\right)$ and $S_{0.5}\left(\alpha_{3}\right)=S_{0.5}\left(\alpha_{4}\right)$. For ranking these intuitionistic fuzzy numbers, we consider the preference attitudinal accuracy values of $\alpha_{1}, \alpha_{2}, \alpha_{3}$, and $\alpha_{4}$ as follows:

$$
\begin{aligned}
& A_{0.5}\left(\alpha_{1}\right)=0.43, \\
& A_{0.5}\left(\alpha_{2}\right)=0.47, \\
& A_{0.5}\left(\alpha_{3}\right)=0.45, \\
& A_{0.5}\left(\alpha_{4}\right)=0.48 .
\end{aligned}
$$

From Definition 26, we have $\alpha_{1} \prec_{0.5} \alpha_{2}$ and $\alpha_{3} \prec_{0.5} \alpha_{4}$. In sum, we have the following ranking order in two cases:

(a) If $\lambda \in[0,0.5)$, then $\alpha_{4} \prec \alpha_{3} \prec \alpha_{2} \prec \alpha_{1}$.

(b) If $\lambda \in[0.5,1]$, then $\alpha_{3} \prec \alpha_{4} \prec \alpha_{1} \prec \alpha_{2}$.

For comparison, we use Xu's method [21], Liu and Wang's method [24], Wang et al's method [22], Lin et al's method [35], and Ye's methods [9] to rank the four intuitionistic fuzzy numbers. The comparative results are listed in Table 2. 
TABLE 2: Comparative results of different methods.

\begin{tabular}{lcc}
\hline Method & Attitudinal parameter & Ranking \\
\hline Xu [21] & Null & $\alpha_{3} \prec \alpha_{4} \prec \alpha_{1} \prec \alpha_{2}$ \\
Liu and Wang [24] & Null & $\alpha_{3} \prec \alpha_{4} \prec \alpha_{1} \prec \alpha_{2}$ \\
Wang et al. [22] & Null & $\alpha_{3} \prec \alpha_{4} \prec \alpha_{1} \prec \alpha_{2}$ \\
Lin et al. [35] & Null & $\alpha_{1} \prec \alpha_{3} \prec \alpha_{2} \prec \alpha_{4}$ \\
Ye [23] & Null & $\alpha_{4} \prec \alpha_{3} \prec \alpha_{2} \prec \alpha_{1}$ \\
Ye [9] & Null & $\alpha_{3} \prec \alpha_{1} \prec \alpha_{4} \prec \alpha_{2}$ \\
Proposed & $\lambda \in[0,0.5)$ & $\alpha_{4} \prec \alpha_{3} \prec \alpha_{2} \prec \alpha_{1}$ \\
Proposed & $\lambda \in[0.5,1]$ & $\alpha_{3} \prec \alpha_{4} \prec \alpha_{1} \prec \alpha_{2}$ \\
\hline
\end{tabular}

From Table 2, we observe that our result is similar to those of Ye's method [23] and differs from those of other methods when $\lambda \in[0,0.5)$. On the other hand, when $\lambda \epsilon$ $[0.5,1]$, our result is similar to those of Xu's method [21], Liu and Wang's method [24], and Wang et al's method [22] and differs from those of other methods. We need to notice that the membership degree of $\alpha_{1}$ is equal to $\alpha_{4}$, and the nonmembership degree of $\alpha_{1}$ is smaller than $\alpha_{4}$. Clearly, the ranking result of $\alpha_{1}$ and $\alpha_{4}$ should be $\alpha_{4} \prec \alpha_{1}$. However, the result is $\alpha_{1} \prec \alpha_{4}$ in Lin et al. [35] and Ye [9], which is illogical and unreasonable. A valid method should be chosen in accordance with the preference attitude of decision maker. The proposed method considers the influence of preference attitude on ranking results comprehensively and makes the ranking results more flexible than other methods.

For ranking the intuitionistic fuzzy numbers in a proper way, the decision maker's preference has been considered in score function. Chen [32] proposed a novel score function by using an attitudinal parameter $\lambda$ for ranking intuitionistic fuzzy numbers. Given an intuitionistic fuzzy number $\alpha=$ $(\mu, v)$, Chen's score function $S_{\lambda}^{C}(\alpha)$ is shown as follows:

$$
S_{\lambda}^{C}(\alpha)=\lambda \mu+(1-\lambda)(1-v), \quad \lambda \in[0,1] .
$$

$0.5<\lambda \leq 1$ and $0 \leq \lambda<0.5$ denote the decision maker's attitude of optimism and pessimism, respectively. When $\lambda=$ 0.5 , the preference attitude of decision maker is indifferent.

By (73), the score values of $\alpha_{1}, \alpha_{2}, \alpha_{3}$, and $\alpha_{4}$ are calculated as

$$
\begin{gathered}
S_{\lambda}^{C}\left(\alpha_{1}\right)=0.8-0.4 \lambda, \\
S_{\lambda}^{C}\left(\alpha_{2}\right)=0.7-0.2 \lambda, \\
S_{\lambda}^{C}\left(\alpha_{3}\right)=0.6-0.3 \lambda, \\
S_{\lambda}^{C}\left(\alpha_{4}\right)=0.5-0.1 \lambda .
\end{gathered}
$$

Since $\lambda \in[0,1]$, we have $S_{\lambda}^{C}\left(\alpha_{3}\right)<S_{\lambda}^{C}\left(\alpha_{1}\right), S_{\lambda}^{C}\left(\alpha_{3}\right)<$ $S_{\lambda}^{C}\left(\alpha_{2}\right)$, and $S_{\lambda}^{C}\left(\alpha_{4}\right)<S_{\lambda}^{C}\left(\alpha_{2}\right)$. However, we notice that when $\lambda=1$, the equality $S_{\lambda}^{C}\left(\alpha_{1}\right)=S_{\lambda}^{C}\left(\alpha_{4}\right)$ holds. That is to say, the order relation between intuitionistic fuzzy numbers $\alpha_{1}$ and $\alpha_{4}$ cannot be determined by Chen's score function. The dynamic score values of Chen's method are shown in Figure 4. Chen's method just considers the extreme points of feasible domain $D_{\alpha}$, but the shape of $D_{\alpha}$ and the other

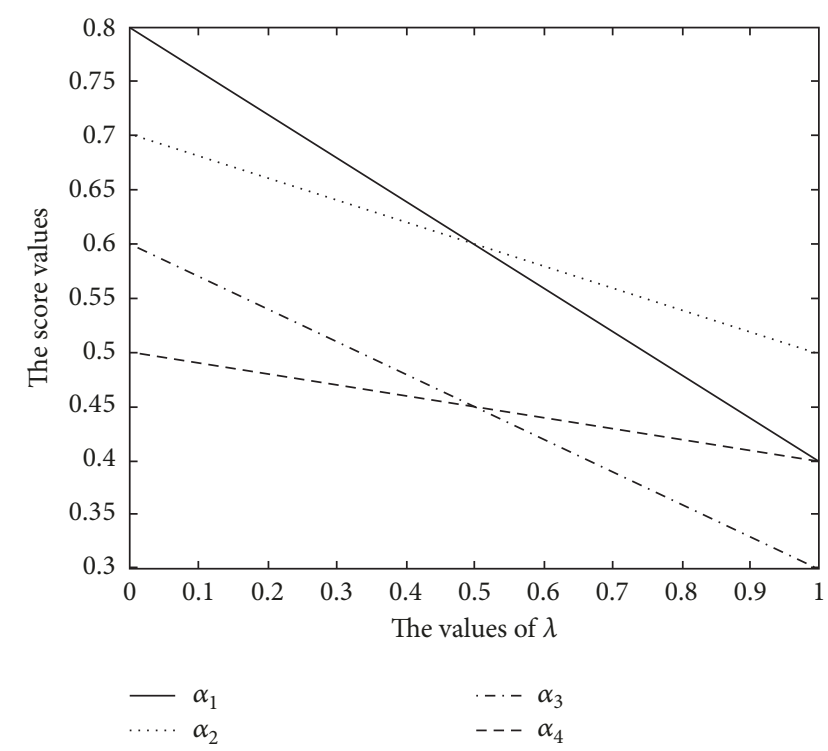

FIGURE 4: The dynamic score values of Chen's method.

possible values in $D_{\alpha}$ are ignored. Thus, it may lose some valuable information, which can be useful in determining the order relation of intuitionistic fuzzy numbers. Therefore, Chen's method for ranking intuitionistic fuzzy numbers is illogical and invalid in some special cases. In the previous calculations, by using the proposed score function, we obtain the ranking result $\alpha_{4} \prec_{\lambda} \alpha_{1}$. The proposed method satisfies some desirable properties and consider all the possible values in $D_{\alpha}$; the ranking of intuitionistic fuzzy numbers can be obtained effectively.

\section{Conclusion and Further Study}

Ranking the intuitionistic fuzzy numbers plays an important role in practical applications under intuitionistic fuzzy settings. This paper proposes a novel ranking method for intuitionistic fuzzy numbers based on the preference attitudinal accuracy and score functions. Meanwhile, some desirable properties of the proposed accuracy and score functions are studied. According to the preference attitude of decision maker, a total order on the set of intuitionistic fuzzy numbers is established. The main advantages of this paper are shown as follows: 
(1) The proposed accuracy and score functions take the preference attitude of decision maker into account and make the ranking results more flexible than other methods.

(2) The preference attitudinal accuracy and score functions consider the shape of $D_{\alpha}$ and all possible values in $D_{\alpha}$, hence avoiding the loss of information in ranking process.

(3) The proposed order relation satisfies irreflexivity, asymmetry, and transitivity. It means that a total order on the set of intuitionistic fuzzy numbers is established.

(4) When $\lambda=0.5$, the proposed order relation is equivalent to Xu's method in Definition 4. That is to say, Xu's method is a special case of the proposed method.

Moreover, we apply the preference attitudinal accuracy and score functions to deal with the renewable energy selection problem under intuitionistic fuzzy environment. In the following research, we intend to make further extensions of the proposed method for ranking other types of fuzzy information. We will consider several applications of the proposed ranking method in actual fields such as pattern recognition, medical diagnosis, and economic forecast.

\section{Disclosure}

Fanyong Meng current affiliation is as follows: Collaborative Innovation Center on Forecast and Evaluation of Meteorological Disasters, Nanjing University of Information Science and Technology, Nanjing 210044, China.

\section{Conflicts of Interest}

The authors declare that they have no conflicts of interest.

\section{Acknowledgments}

This work was supported by the National Natural Science Foundation of China (no. 71601049), the Humanities and Social Sciences Fund of the Ministry of Education (no. 16YJC630064), the Natural Science Foundation of Fujian Province (no. 2016J01282), and the University Training Program in Scientific Research for Outstanding Young Talents of Fujian Province.

\section{References}

[1] K. T. Atanassov, "Intuitionistic fuzzy sets," Fuzzy Sets and Systems, vol. 20, no. 1, pp. 87-96, 1986.

[2] S. Zeng and J. Chen, "Intuitionistic fuzzy decision making based on OWA and distance measures," Communications in Computer and Information Science, vol. 308, no. 2, pp. 243-248, 2012.

[3] J. Wu and F. Chiclana, "A risk attitudinal ranking method for interval-valued intuitionistic fuzzy numbers based on novel attitudinal expected score and accuracy functions," Applied Soft Computing, vol. 22, pp. 272-286, 2014.
[4] Z. Pei, "Intuitionistic fuzzy variables: concepts and applications in decision making," Expert Systems with Applications, vol. 42, no. 22, pp. 9033-9045, 2015.

[5] H. Liao, Z. Xu, X. J. Zeng, and D. L. Xu, "An enhanced consensus reaching process in group decision making with intuitionistic fuzzy preference relations," in Proceedings of the Information Sciences An International Journal, vol. 329, pp. 274-286, 2016.

[6] H. Garg, "Novel intuitionistic fuzzy decision making method based on an improved operation laws and its application," Engineering Applications of Artificial Intelligence, vol. 60, pp. 164-174, 2017.

[7] Z. Hao, Z. Xu, H. Zhao, and R. Zhang, "Novel intuitionistic fuzzy decision making models in the framework of decision field theory," Information Fusion, vol. 33, pp. 57-70, 2017.

[8] F. Ye, "An extended TOPSIS method with interval-valued intuitionistic fuzzy numbers for virtual enterprise partner selection," Expert Systems with Applications, vol. 37, no. 10, pp. 7050-7055, 2010.

[9] J. Ye, "Multicriteria fuzzy decision-making method using entropy weights-based correlation coefficients of intervalvalued intuitionistic fuzzy sets," Applied Mathematical Modelling, vol. 34, no. 12, pp. 3864-3870, 2010.

[10] J. Ashayeri, G. Tuzkaya, and U. R. Tuzkaya, "Supply chain partners and configuration selection: An intuitionistic fuzzy Choquet integral operator based approach," Expert Systems with Applications, vol. 39, no. 3, pp. 3642-3649, 2012.

[11] D. F. Li and C. T. Cheng, "New similarity measures of intuitionistic fuzzy sets and application to pattern recognitions," Pattern Recognition Letters, vol. 23, no. 1, pp. 221-225, 2002.

[12] H. B. Mitchell, "On the Dengfeng-Chuntian similarity measure and its application to pattern recognition," Pattern Recognition Letters, vol. 24, no. 16, pp. 3101-3104, 2003.

[13] Y. Song, X. Wang, L. Lei, and A. Xue, "A novel similarity measure on intuitionistic fuzzy sets with its applications," Applied Intelligence, vol. 42, no. 2, pp. 252-261, 2015.

[14] F. Meng and X. Chen, "Entropy and similarity measure of Atanassov's intuitionistic fuzzy sets and their application to pattern recognition based on fuzzy measures," Pattern Analysis and Applications, vol. 19, no. 1, pp. 11-20, 2016.

[15] S. K. De, R. Biswas, and A. R. Roy, "An application of intuitionistic fuzzy sets in medical diagnosis," Fuzzy Sets and Systems, vol. 117, no. 2, pp. 209-213, 2001.

[16] H. S. Le and N. T. Thong, "Intuitionistic fuzzy recommender systems: an effective tool for medical diagnosis," KnowledgeBased Systems, vol. 74, no. 1, pp. 133-150, 2015.

[17] R. Saadati, S. M. Vaezpour, and Y. J. Cho, "Quicksort algorithm: Application of a fixed point theorem in intuitionistic fuzzy quasi-metric spaces at a domain of words," Journal of Computational and Applied Mathematics, vol. 228, no. 1, pp. 219225, 2009.

[18] Q. Zhang, "Study on the software quality evaluation based on artificial intelligence with intuitionistic fuzzy information," International Journal of Digital Content Technology and its Applications, vol. 6, no. 22, pp. 371-376, 2012.

[19] S. M. Chen and J. M. Tan, "Handling multicriteria fuzzy decision-making problems based on vague set theory," Fuzzy Sets and Systems, vol. 67, no. 2, pp. 163-172, 1994.

[20] D. H. Hong and C. H. Choi, "Multicriteria fuzzy decisionmaking problems based on vague set theory," Fuzzy Sets and Systems, vol. 114, no. 1, pp. 103-113, 2000. 
[21] Z. Xu, "Intuitionistic fuzzy aggregation operators," IEEE Transactions on Fuzzy Systems, vol. 15, no. 6, pp. 1179-1187, 2007.

[22] J. Wang, J. Zhang, and S. Y. Liu, "A new score function for fuzzy MCDM based on vague set theory," in Proceedings of the International Journal of Computational Cognition, vol. 4, pp. 4448, 2006.

[23] J. Ye, "Improved method of multicriteria fuzzy decision-making based on vague sets," Computer-Aided Design, vol. 39, no. 2, pp. 164-169, 2007.

[24] H. Liu and G. Wang, "Multi-criteria decision-making methods based on intuitionistic fuzzy sets," European Journal of Operational Research, vol. 179, no. 1, pp. 220-233, 2007.

[25] E. Jafarian and M. A. Rezvani, "A valuation-based method for ranking the intuitionistic fuzzy numbers," Journal of Intelligent \& Fuzzy Systems: Applications in Engineering and Technology, vol. 24, no. 1, pp. 133-144, 2013.

[26] X. Yu, Z. Xu, S. Liu, and Q. Chen, "On ranking of intuitionistic fuzzy values based on dominance relations," International Journal of Uncertainty, Fuzziness and Knowledge-Based Systems, vol. 22, no. 2, pp. 315-335, 2014.

[27] K. Guo, "Amount of information and attitudinal-based method for ranking atanassov's intuitionistic fuzzy values," IEEE Transactions on Fuzzy Systems, vol. 22, no. 1, pp. 177-188, 2014.

[28] Z. Zhang and Z. Xu, "The orders of intuitionistic fuzzy numbers," Journal of Intelligent and Fuzzy Systems, vol. 28, pp. 505511, 2015.

[29] G. N. V. Lakshmana, S. Jeevaraj, and S. Geetha, "Total ordering for intuitionistic fuzzy numbers," Complexity, vol. 21, no. S2, pp. 54-66, 2016.

[30] P. Gupta, C.-T. Lin, M. K. Mehlawat, and N. Grover, "A New Method for Intuitionistic Fuzzy Multiattribute Decision Making," IEEE Transactions on Systems, Man, and Cybernetics: Systems, vol. 46, no. 9, pp. 1167-1179, 2016.

[31] Z. Xing, W. Xiong, and H. Liu, "A Euclidean approach for ranking atanassov intuitionistic fuzzy values," Transactions on Fuzzy Systems, vol. 99, pp. 1-13, 2017.

[32] T.-Y. Chen, "A comparative analysis of score functions for multiple criteria decision making in intuitionistic fuzzy settings," Information Sciences, vol. 181, no. 17, pp. 3652-3676, 2011.

[33] J.-Q. Wang, K.-J. Li, H.-Y. Zhang, and X.-H. Chen, "A score function based on relative entropy and its application in intuitionistic normal fuzzy multiple criteria decision making," Journal of Intelligent \& Fuzzy Systems: Applications in Engineering and Technology, vol. 25, no. 3, pp. 567-576, 2013.

[34] S.-P. Wan, F. Wang, and J.-Y. Dong, "A novel risk attitudinal ranking method for intuitionistic fuzzy values and application to MADM," Applied Soft Computing, vol. 40, pp. 98-112, 2016.

[35] L. Lin, X.-H. Yuan, and Z.-Q. Xia, "Multicriteria fuzzy decisionmaking methods based on intuitionistic fuzzy sets," Journal of Computer and System Sciences, vol. 73, no. 1, pp. 84-88, 2007.

[36] Q. Qin, F. Liang, L. Li, Y.-W. Chen, and G.-F. Yu, "A TODIMbased multi-criteria group decision making with triangular intuitionistic fuzzy numbers," Applied Soft Computing, vol. 55, pp. 93-107, 2017. 


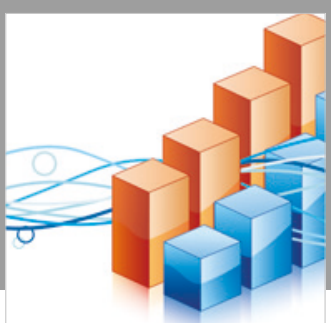

Advances in

Operations Research

\section{-n-m}
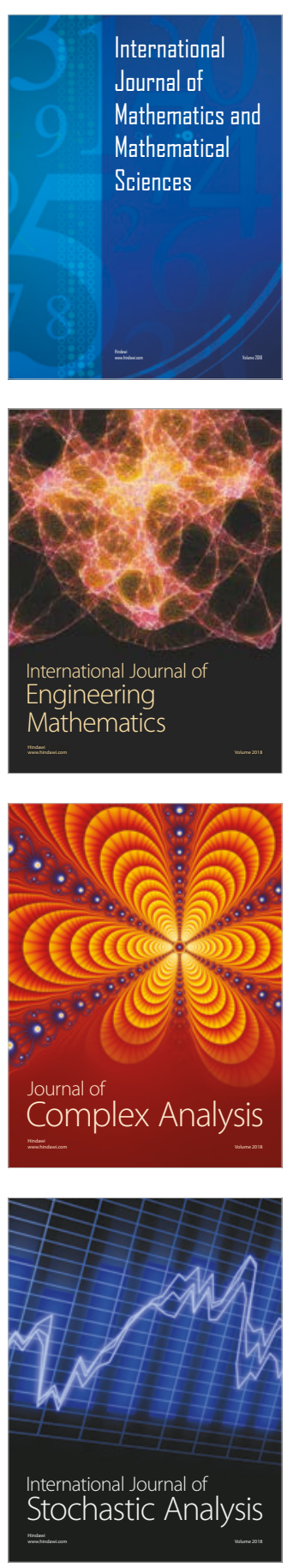
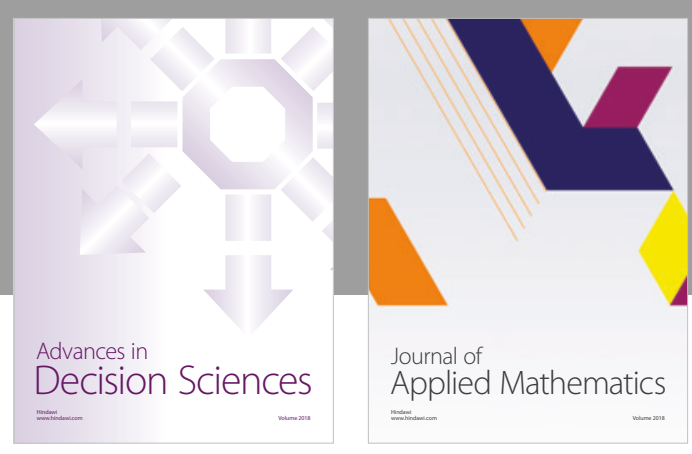

Journal of

Applied Mathematics
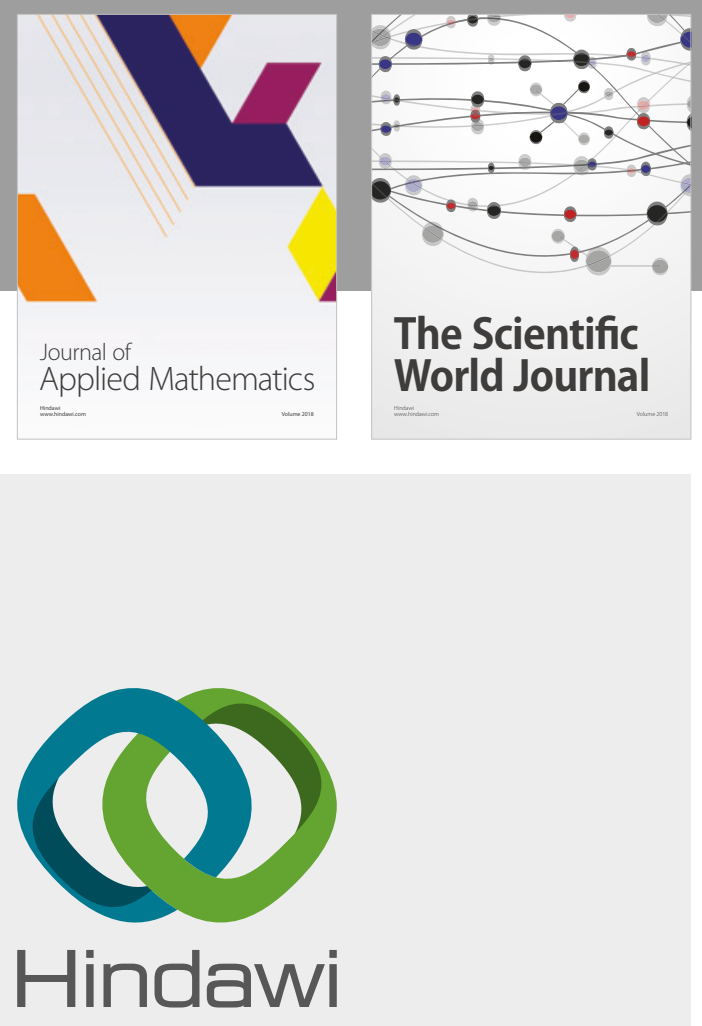

Submit your manuscripts at

www.hindawi.com

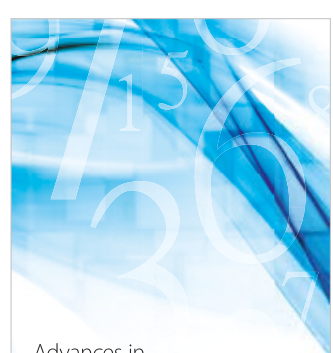

Advances in
Numerical Analysis
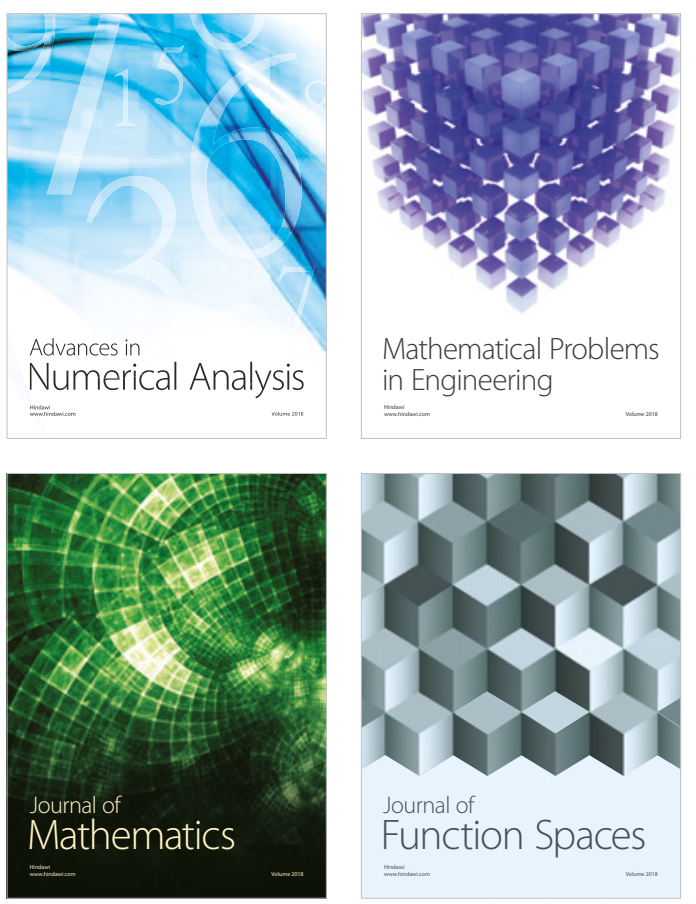

Mathematical Problems in Engineering

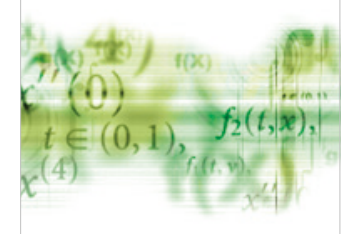

International Journal of

Differential Equations

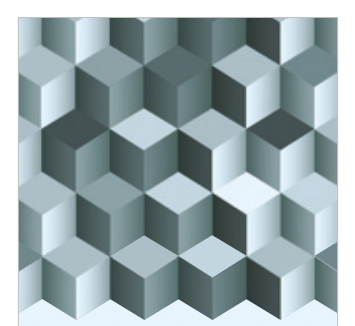

Journal of

Function Spaces
The Scientific

World Journal

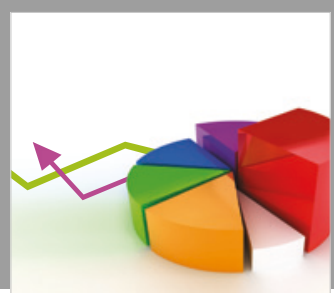

Journal of

Probability and Statistics
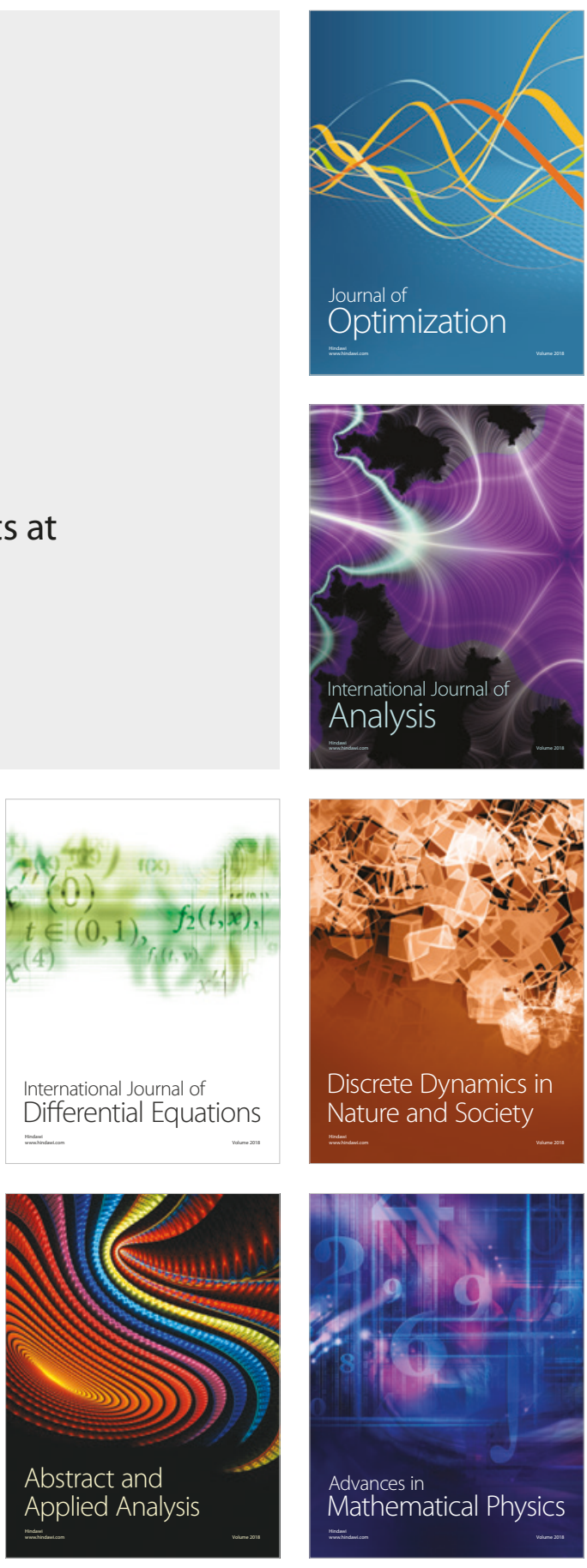